\title{
Potential scalp stimulation targets for mental disorders: evidence from neuroimaging studies
}

\author{
Jin Cao, Thalia Celeste Chai-Zhang, Yiting Huang, Maya Nicole Eshel and Jian Kong (1)
}

\begin{abstract}
Mental disorders widely contribute to the modern global disease burden, creating a significant need for improvement of treatments. Scalp stimulation methods (such as scalp acupuncture and transcranial electrical stimulation) have shown promising results in relieving psychiatric symptoms. However, neuroimaging findings haven't been wellintegrated into scalp stimulation treatments. Identifying surface brain regions associated with mental disorders would expand target selection and the potential for these interventions as treatments for mental disorders. In this study, we performed large-scale meta-analyses separately on eight common mental disorders: attention deficit hyperactivity disorder, anxiety disorder, autism spectrum disorder, bipolar disorder, compulsive disorder, major depression, posttraumatic stress disorder and schizophrenia; utilizing modern neuroimaging literature to summarize disorder-associated surface brain regions, and proposed neuroimaging-based target protocols. We found that the medial frontal gyrus, the supplementary motor area, and the dorsal lateral prefrontal cortex are commonly involved in the pathophysiology of mental disorders. The target protocols we proposed may provide new brain targets for scalp stimulation in the treatment of mental disorders, and facilitate its clinical application.
\end{abstract}

Keywords: Neuroimaging, Meta-analysis, Scalp stimulation, Scalp acupuncture, Transcranial electrical stimulation, Mental disorder

\section{Introduction}

Mental disorders are a major component of the modern global disease burden. However, the quality of the therapeutic outcomes of pharmacologic treatments for mental disorders is ambiguous due to their side effects, withdrawal symptoms, and risk of abuse. Thus, increasing attention has been given towards non-pharmacological interventions over recent years.

Recently, scalp stimulation methods, i.e., applying transcranial stimulation on the scalp to modulate the function of corresponding brain areas to relive symptoms, have drawn increased attention of investigators. Scalp stimulation methods may include many different current treatments.

*Correspondence: jkong2@mgh.harvard.edu

Department of Psychiatry, Massachusetts General Hospital, Harvard

Medical School, Charlestown, MA 02129, USA
For instance, scalp acupuncture, a modern school of acupuncture developed on the basis of anatomical and neurophysiological knowledge [1], may be considered as an early scalp stimulation treatment. Scalp acupuncture aims to modulate certain brain areas, thereby providing therapeutic benefits for a wide scope of diseases through the stimulation of specific scalp areas corresponding to certain cortical areas. Scalp acupuncture may be applied by manual stimulation or electrical stimulation (similar to transcranial alternating current stimulation [tACS]). Accumulating evidence has demonstrated the potential of scalp acupuncture in relieving symptoms of mental disorders $[2,3]$.

Nevertheless, current prescriptions of scalp acupuncture for varying disorders are mainly based on an understanding of brain functions from the 1970s, when the practice was first introduced. In recent decades, there has been remarkable progress made in original author(s) and the source, provide a link to the Creative Commons licence, and indicate if changes were made. The images or other third party material in this article are included in the article's Creative Commons licence, unless indicated otherwise in a credit line to the material. If material is not included in the article's Creative Commons licence and your intended use is not permitted by statutory regulation or exceeds the permitted use, you will need to obtain permission directly from the copyright holder. To view a copy of this licence, visit http://creativecommons.org/licenses/by/4.0/. The Creative Commons Public Domain Dedication waiver (http://creativeco mmons.org/publicdomain/zero/1.0/) applies to the data made available in this article, unless otherwise stated in a credit line to the data. 
understanding the neural circuitry of mental disorders through cutting-edge brain imaging techniques [4-6]. Unfortunately, these advances have yet to be incorporated into scalp acupuncture treatments for mental disorders.

Modern brain stimulation methods such as transcranial electrical stimulation (tES) may also be considered scalp stimulation methods. For example, transcranial direct current stimulation (tDCS), a form of $\mathrm{tES}$, is based on the application of a weak, direct electric current delivered over the scalp to induce polarity dependent changes in cortical excitability (anodal and cathodal stimulation induce increasing and decreasing cortical excitability, respectively), and has shown promising results in ameliorating the symptoms of mental disorders [7-9]. Another method, transcranial alternating current stimulation (tACS), a different form of tES, can modulate the rhythms of endogenous oscillations by applying weak alternating current through the scalp, and has also demonstrated potential in alleviating anxiety, depression, compulsive disorders, etc. [10-12].

Literature suggests that multiple brain regions/networks are involved in the pathophysiology of mental disorders. Yet as of current date, many tES studies have only used the prefrontal cortex as the target area for stimulation [13, 14]. Identifying other brain regions associated with mental disorders (particularly among surface brain areas which are suitable for $\mathrm{tES}$ ) would expand target selection and may represent a crucial step to increasing the potential for neuromodulation techniques as treatment for mental disorders.

In recent decades, the development of brain neuroimaging techniques has spurred rapid growth of literature on human brain imaging studies of mental disorders and has significantly advanced our understanding of the complex brain pathophysiology associated with these disorders. However, the rich literature also introduces new challenges, i.e., we are now burdened with an excess of data to work through. It has therefore become necessary to develop new techniques for the large-scale aggregation and synthesis of human neuroimaging data [15].

Neurosynth is a new brain mapping framework that can incorporate text-mining, meta-analysis and machinelearning techniques to generate probabilistic mappings between mental disorders and neural states that can be used for a broad range of neuroimaging applications and generate large-scale meta-analyses for hundreds of broad psychological concepts. Previous approaches have relied heavily on researchers' manual efforts or specific domains/focuses (for example: resting state functional connectivity, task related fMRI associated with a specific disorder), which may have limited the scope and efficiency of resulting analyses [15].
Thus, with the aid of Neurosynth, this study aims to develop neuroimaging-based target protocols for eight common mental disorders (attention deficit hyperactivity disorder (ADHD), anxiety disorder, autism spectrum disorder (ASD), bipolar disorder, compulsive disorder, major depression, post-traumatic stress disorder (PTSD) and schizophrenia) for tES, scalp acupuncture, repetitive transcranial magnetic stimulation (rTMS) $[9,14,16]$, focused ultrasound (FUS) [17] and other brain stimulation methods when applicable. Specifically, we first used Neurosynth to generate large-scale meta-analyses for the above eight mental disorders. Then, we further refined/ simplified the findings from the neuroimaging analyses, and proposed neuroimaging-based scalp stimulation target protocol for each disorder to facilitate its clinical application. We hypothesize that different mental disorders will be associated with distinguishable scalp stimulation targets, although there may be common/overlapping targets across different disorders.

\section{Methods}

To identify disorder-associated brain regions, we used Neurosynth (http://neurosynth.org/:accessed21Septe mber2020) as a metadata reference for neuroimaging literature. In this study, we applied a Bayesian reverse inference term-based meta-analytic approach from Neurosynth that extracted data from all published neuroimaging studies included in the Neurosynth database. This method is different from the classic forward inference that selected voxels for inclusion in a given map based on their positive association with each mental disorder, and instead also included all negative findings thus allowing for greater specificity [15]. Under the search string "disorder name" (i.e., search strings "ADHD", "anxiety disorder", "autism spectrum", "bipolar disorder", "compulsive disorder", "major depression", "PTSD", and "schizophrenia"), neuroimaging studies were identified, and a uniformity test map was generated to identify disorder-associated brain regions. Complete lists of the studies included for each disorder extracted from Neurosynth can be found in Additional file 1: Tables S1-S8.

Since scalp stimulations such as scalp acupuncture and tES will predominantly influence the surface brain areas, similar to our previous studies [18-21], a brain surface cortical mask was created using the SPM Wake Forest University (WFU) PickAtlas toolbox (http://fmri. wfubmc.edu/software/pickatlas, version 3.0.5) to identify disorder-associated surface brain regions [18-21]. Next, brain regions from the meta-analysis were refined by discerning the overlap of the uniformity test map with the brain surface cortical masks and then using the xjView toolbox (http://www.alivelearn.net/xjview) to identify the coordinates with peak z-scores within the all-surface 
cluster larger than 30 voxels on the uniformity test map (Additional file 1: Figure S1).

The results from the meta-analysis were mapped onto a standard brain using SurfIce (https://www.nitrc.org/proje cts/surfice) and a standard head using MRIcroGL (http:// www.mccauslandcenter.sc.edu/mricrogl) with the international 10-20 electroencephalography (EEG) system in MNI space. The MNI coordinates of the 10-20 EEG system were extracted from a previous study [22] (Additional file 1: Figure S1).

To facilitate clinical application, we refined the disorder-associated surface clusters to eight/nine clusters (with the largest cluster size/peak intensity among all clusters) using xjView toolbox and identified peak coordinates of these clusters as the potential scalp stimulation targets for corresponding disorders. Furthermore, 2-mm radius spherical masks centered on the identified peak coordinates were created using WFU_PickAtlas toolbox and mapped onto a standard brain using SurfIce with the international 10-20 EEG system in MNI space for indicating the locations. In addition, we applied the international standard acupoints to facilitate identifying the locations. Finally, we visually checked the locations of the brain regions obtained to identify potential brain surface targets that are accessible by scalp acupuncture, tES, and other scalp stimulation methods, and proposed neuroimaging-based scalp stimulation target prescriptions (e.g., ADHD-1 to ADHD-9, ANX-1 to ANX-9, AUT-1 to AUT-9, etc.) for each disorder based on the neuroimaging findings. To help the readers understand the specific brain function of identified areas, we also summarized the brain functions of each identified surface region associated with a corresponding mental disorder (functions were collected and summarized based on https://neuro synth.org and http://www.fmriconsulting.com/brodm ann) (Additional file 1: Figure S1).

Since mental disorders are conceived as an interconnected system of symptoms in which certain symptoms are the cause of others and can be dominant in specific brain regions; we also explored the overlap surface regions among the eight mental disorders using xjView toolbox. Results (with peak coordinates showing on each overlap region) were mapped onto a standard brain using MRIcroGL for demonstration.

\section{Results}

\section{Meta-analysis results}

\section{A. ADHD}

144 studies (extracted from Neurosynth) were included in the data analysis (a complete list of the 144 studies can be found in Additional file 1: Table S1). Twenty-one clusters on the brain surface were identified from the uniformity test map of the meta-analysis (Table 1).
These brain regions were the bilateral middle frontal gyrus (MFG), inferior frontal gyrus (IFG), orbital inferior frontal gyrus (OrbIFG), triangular inferior frontal gyrus (TriIFG), dorsolateral prefrontal cortex (dlPFC), medial prefrontal cortex (mPFC), superior parietal lobule (SPL), inferior parietal lobule (IPL), supramarginal gyrus (SMG) and precuneus $(\mathrm{PCu})$, the left medial frontal gyrus (MedFG), superior medial frontal gyrus (SupMFG), orbital medial frontal gyrus (OrbMFG), angular gyrus (AG), precentral gyrus (PreCG), postcentral gyrus (PoCG), supplementary motor area (SMA), and superior temporal gyrus (STG) and middle temporal gyrus (MTG) (Table 1, Fig. 1A).

\section{B. Anxiety disorder}

95 studies (extracted from Neurosynth) were included in the data analysis (a complete list of the 95 studies can be found in Additional file 1: Table S2). Fourteen clusters on the brain surface were identified from the uniformity test map of the meta-analysis (Table 2).

These surface regions included the left superior frontal gyrus (SFG)/superior medial frontal gyrus (SupMFG), middle temporal gyrus (MTG), and middle occipital gyrus (MOG)/inferior occipital gyrus (IOG); the right dorsolateral prefrontal cortex (dlPFC), supplementary motor area (SMA), superior temporal pole (STP), superior parietal lobule (SPL)/supramarginal gyrus (SMG)/ angular gyrus (AG), precentral gyrus (PreCG), and superior occipital gyrus (SOG); as well as the bilateral inferior frontal gyrus (IFG), middle frontal gyrus (MFG), and inferior frontal operculum (IFO) (Table 2, Fig. 1B).

\section{Autism spectrum}

170 studies (extracted from Neurosynth) were included in the data analysis (a complete list of the 170 studies can be found in Additional file 1: Table S3). Nineteen clusters on the brain surface were identified from the uniformity test map of the meta-analysis (Table 3).

These brain regions were the bilateral middle temporal gyrus (MTG), inferior temporal gyrus (ITG), superior temporal gyrus (STG), superior occipital gyrus (SOG), middle occipital gyrus (MOG), inferior occipital gyru (IOG), superior frontal gyrus (SFG), middle frontal gyrus (MFG), inferior frontal gyrus (IFG), orbital inferior frontal gyrus (OrbIFG), triangular inferior frontal gyrus (TrilFG), opercular inferior frontal gyrus (OperIFG), Rolandic operculum (RO), superior parietal lobule (SPL), inferior parietal lobule (IPL), supramarginal gyrus (SMG), angular gyrus (AG), precentral gyrus (PreCG), postcentral gyrus (PoCG) and precuneus (PCu), the left cuneus, as well as the right superior medial frontal gyrus (SupMFG), medial frontal gyrus (MedFG), orbital medial 
Table 1 Coordinates of ADHD-associated surface regions identified from meta-analysis

\begin{tabular}{|c|c|c|c|c|c|c|}
\hline \multirow[t]{2}{*}{ Cluster ID } & \multirow[t]{2}{*}{ Cluster size } & \multirow[t]{2}{*}{ Peak T } & \multicolumn{3}{|c|}{ Peak coordinates } & \multirow[t]{2}{*}{ Brain regions } \\
\hline & & & $\mathrm{x}$ & y & $\mathbf{z}$ & \\
\hline 1 & 105 & 6.98 & -2 & 50 & -10 & L MedFG/OrbMFG \\
\hline 2 & 79 & 8.62 & 42 & 24 & -8 & RIFG/OrbIFG \\
\hline 3 & 205 & 8.62 & -36 & 22 & -8 & L IFG/OrbIFG \\
\hline 4 & 168 & 6.98 & 36 & 24 & 8 & RIFG/TrilFG \\
\hline 5 & 39 & 4.51 & 58 & -44 & 8 & RSTG/MTG \\
\hline 6 & 39 & 4.51 & -40 & 46 & 10 & L MFG/TrilFG \\
\hline 7 & 55 & 5.33 & -2 & 46 & 18 & L MedFG/SupMFG/dlPFC/mPFC \\
\hline 8 & 35 & 5.33 & 46 & 36 & 24 & R MFG/TrilFG \\
\hline 9 & 67 & 5.33 & -42 & 30 & 18 & L MFG/TrilFG \\
\hline 10 & 66 & 5.33 & -58 & -50 & 28 & LIPL/SMG \\
\hline 11 & 36 & 5.33 & -50 & -64 & 32 & LIPL/AG \\
\hline 12 & 188 & 6.98 & -48 & 10 & 32 & L IFG/dlPFC/mPFC/PreCG \\
\hline 13 & 99 & 6.15 & 58 & -46 & 30 & $\mathrm{RIPL/SMG}$ \\
\hline 14 & 81 & 6.98 & 36 & 26 & 40 & R MFG/dIPFC/mPFC \\
\hline 15 & 71 & 5.33 & 8 & -68 & 36 & R SPL/PCu/cuneus \\
\hline 16 & 120 & 6.98 & -40 & -56 & 46 & L IPL/PCu/AG \\
\hline 17 & 236 & 8.62 & -2 & 20 & 42 & L MedFG/SupMFG/mPFC \\
\hline 18 & 71 & 5.33 & 48 & -46 & 46 & $\mathrm{RIPL} / \mathrm{SMG}$ \\
\hline 19 & 82 & 6.15 & -28 & -54 & 42 & L SPL/IPL/PCu \\
\hline 20 & 36 & 5.33 & -8 & -6 & 52 & L MedFG/SMA \\
\hline 21 & 31 & 5.33 & -42 & -26 & 52 & L SPL/PoCG \\
\hline
\end{tabular}

L, left; R, right; MFG, middle frontal gyrus; IFG, inferior frontal gyrus; OrbIFG, orbital inferior frontal gyrus; SupMFG, superior medial frontal gyrus; MedFG, medial frontal gyrus; OrbMFG, orbital medial frontal gyrus; TrilFG, triangular inferior frontal gyrus; dIPFC, dorsolateral prefrontal cortex; mPFC, medial prefrontal cortex; STG, superior temporal gyrus; MTG, middle temporal gyrus; SPL, superior parietal lobule; IPL, inferior parietal lobule; SMG, supramarginal gyrus; AG, angular gyrus; PreCG, precentral gyrus; PoCG, postcentral gyrus; PCu, precuneus; SMA, supplementary motor area

frontal gyrus (OrbMFG) and supplementary motor area (SMA) (Table 3, Fig. 1C).

\section{Bipolar disorder}

130 studies (extracted from Neurosynth) were included in the data analysis (a complete list of the 130 studies can be found in Additional file 1: Table S4). Eight clusters on the brain surface were identified from the uniformity test map of the meta-analysis (Table 4).

These brain regions were the bilateral inferior frontal gyrus (IFG), superior frontal gyrus (SFG), superior parietal lobule (SPL) and inferior parietal lobule (IPL), the left orbital inferior frontal gyrus (OrbIFG), superior temporal pole (STP), medial frontal gyrus (MedFG), superior temporal gyrus (STG) and supplementary motor area (SMA), as well as the right middle frontal gyrus (MFG), middle occipital gyrus (MOG), inferior occipital gyrus (IOG), inferior temporal gyrus (ITG), opercular inferior frontal gyrus (OperIFG), SMG, supramarginal gyrus (SMG), angular gyrus (AG) and precentral gyrus (PreCG) (Table 4, Fig. 1D).

\section{E. Compulsive disorder}

92 studies (extracted from Neurosynth) were included in the data analysis (a complete list of the 92 studies can be found in Additional file 1: Table S5). Seventeen clusters on the brain surface were identified from the uniformity test map of the meta-analysis (Table 5).

These brain regions were the bilateral middle frontal gyrus (MFG), inferior frontal gyrus (IFG), orbital inferior frontal gyrus (OrbIFG), triangular inferior frontal gyrus (TriIFG), opercular inferior frontal gyrus (OperIFG), superior temporal gyrus (STG), middle temporal gyrus (MTG), inferior temporal gyrus (ITG), precentral gyrus (PreCG), postcentral gyrus (PoCG), Rolandic operculum (RO), middle occipital gyrus (MOG) and inferior occipital gyrus (IOG), the left superior frontal gyrus (SFG), superior medial frontal gyrus (SupMFG), medial frontal gyrus (MedFG), superior temporal pole (STP), superior parietal lobule (SPL), inferior parietal lobule (IPL), supramarginal gyrus (SMG), angular gyrus (AG), precuneus $(\mathrm{PCu})$, supplementary motor area (SMA), as well as the right cuneus (Table 5, Fig. 1E). 


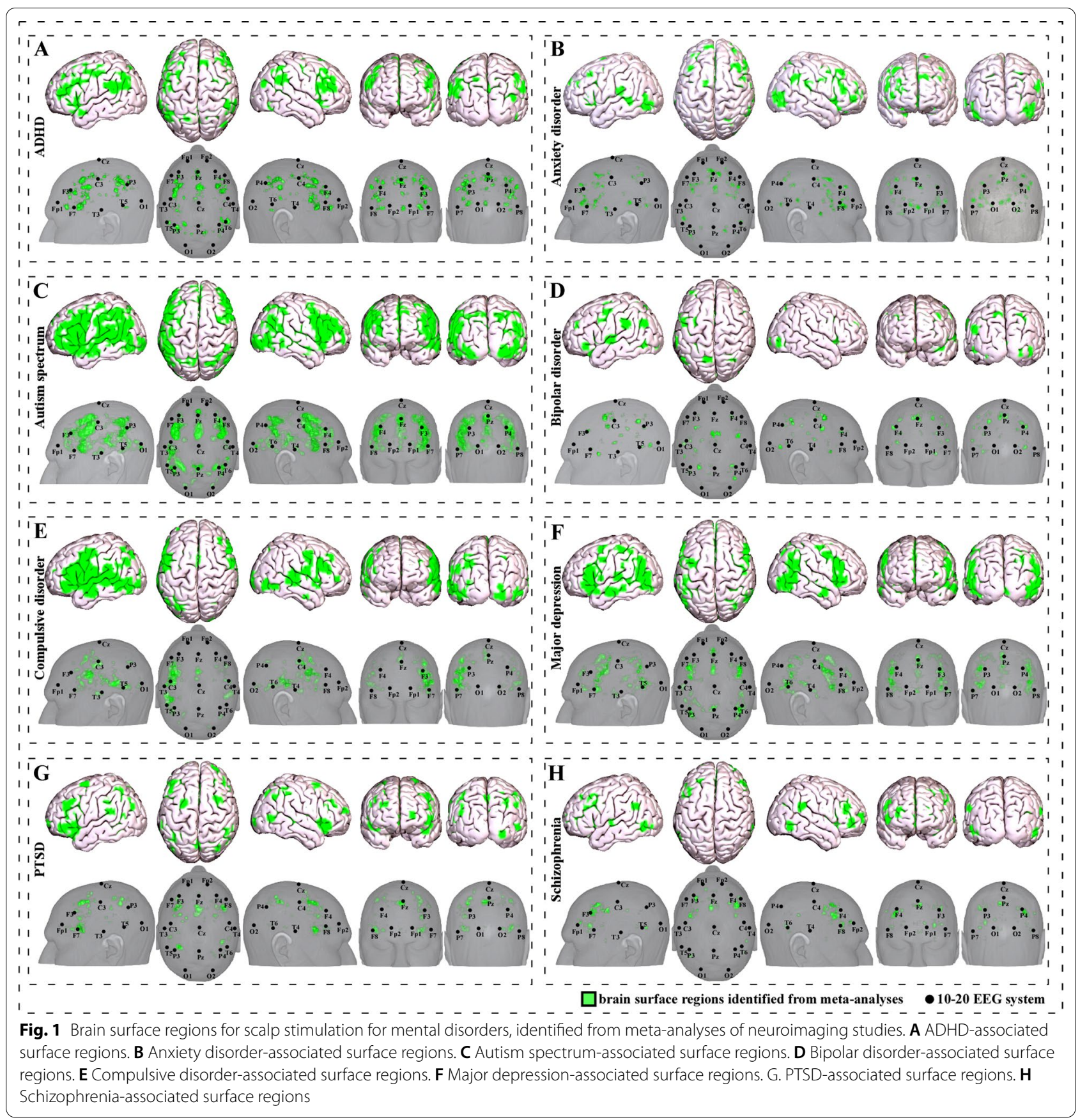

\section{F. Major depression}

77 studies (extracted from Neurosynth) were included in the data analysis (a complete list of the 77 studies can be found in Additional file 1: Table S6). Eighteen clusters on the brain surface were identified from the uniformity test map of the meta-analysis (Table 6).

These brain regions were the bilateral middle frontal gyrus (MFG), inferior frontal gyrus (IFG), orbital inferior frontal gyrus (OrbIFG), triangular inferior frontal gyrus
(TriIFG), opercular inferior frontal gyrus (OperIFG), superior temporal gyrus (STG), middle temporal gyrus (MTG), inferior temporal gyrus (ITG), inferior parietal lobule (IPL), angular gyrus (AG), precentral gyrus (PreCG), postcentral gyrus (PoCG), precuneus $(\mathrm{PCu})$ and middle occipital gyrus (MOG), the left superior frontal gyrus (SFG), medial frontal gyrus (MedFG), superior medial frontal gyrus (SupMFG), orbital medial frontal gyrus (OrbMFG), superior parietal lobule (SPL) and 
Table 2 Coordinates of anxiety-associated surface regions identified from meta-analysis

\begin{tabular}{|c|c|c|c|c|c|c|}
\hline \multirow[t]{2}{*}{ Cluster ID } & \multirow[t]{2}{*}{ Cluster size } & \multirow[t]{2}{*}{ Peak T } & \multicolumn{3}{|c|}{ Peak coordinates } & \multirow[t]{2}{*}{ Brain regions } \\
\hline & & & $\mathbf{x}$ & $y$ & $\mathbf{z}$ & \\
\hline 1 & 55 & 12.80 & 28 & 4 & -22 & R STP \\
\hline 2 & 178 & 6.88 & -32 & 26 & -6 & LIFG/OrblFG \\
\hline 3 & 276 & 6.88 & -2 & 54 & -12 & L MedFG/OrbMFG \\
\hline 4 & 55 & 6.88 & -44 & -74 & -8 & LMOG/IOG \\
\hline 5 & 80 & 6.88 & -56 & -40 & 2 & L MTG \\
\hline 6 & 30 & 7.87 & 46 & 10 & 4 & RIFO \\
\hline 7 & 101 & 5.89 & 50 & 22 & 20 & $\mathrm{R} I F G / \mathrm{d} I \mathrm{PFC}$ \\
\hline 8 & 61 & 5.89 & -6 & 56 & 28 & LSFG/SupMFG \\
\hline 9 & 40 & 5.89 & -38 & 14 & 32 & LMFG/IFO \\
\hline 10 & 47 & 5.89 & 56 & -46 & 34 & R SPL/SMG/AG \\
\hline 11 & 53 & 5.89 & 42 & 0 & 46 & R MFG/PreCG \\
\hline 12 & 112 & 7.86 & 2 & 18 & 44 & RSMA \\
\hline 13 & 55 & 4.90 & 38 & 28 & 42 & RMFG \\
\hline 14 & 51 & 4.90 & 24 & -66 & 44 & R SPL/SOG \\
\hline
\end{tabular}

L, left; R, right; STP, superior temporal pole; IFG, inferior frontal gyrus; OrbIFG, orbital inferior frontal gyrus; MedFG, medial frontal gyrus; OrbMFG, orbital medial frontal gyrus; MOG, middle occipital gyrus; IOG, inferior occipital gyrus; MTG, middle temporal gyrus; IFO, inferior frontal operculum; dIPFC, dorsolateral prefrontal cortex; SFG, superior frontal gyrus; SupMFG, superior medial frontal gyrus; MFG, middle frontal gyrus; SPL, superior parietal lobule; SMG, supramarginal gyrus; AG, angular gyrus; PreCG, precentral gyrus; SMA, supplementary motor area; SOG, superior occipital gyrus

Table 3 Coordinates of autism spectrum disorder-associated surface regions identified from meta-analysis

\begin{tabular}{|c|c|c|c|c|c|c|}
\hline \multirow[t]{2}{*}{ Cluster ID } & \multirow[t]{2}{*}{ Cluster size } & \multirow[t]{2}{*}{ Peak T } & \multicolumn{3}{|c|}{ Peak coordinates } & \multirow[t]{2}{*}{ Brain regions } \\
\hline & & & $\mathbf{x}$ & y & z & \\
\hline 1 & 125 & 5.97 & -56 & -6 & -16 & L MTG/ITG/STG \\
\hline 2 & 109 & 5.97 & 58 & -6 & -16 & $\mathrm{RMTG} / \mathrm{ITG} / \mathrm{STG}$ \\
\hline 3 & 2515 & 17.27 & -46 & 10 & 32 & L IFG/TrilFG/OrbIFG/OperlFG/MFG/PreCG/STG/RO \\
\hline 4 & 74 & 7.03 & 44 & -54 & -16 & R ITG/MOG \\
\hline 5 & 2334 & 11.62 & -30 & -56 & 40 & L IPL/SPL/STG/MTG/PreCG/PoCG/RO/SMG/AG/MOG \\
\hline 6 & 2160 & 17.27 & 34 & 26 & -6 & R IFG/OrbIFG/OperlFG/TrilFG/MFG/PreCG \\
\hline 7 & 467 & 7.38 & 0 & 54 & 24 & SupMFG/MedFG/OrbMFG/ \\
\hline 8 & 102 & 6.32 & -22 & -94 & -4 & L IOG/MOG/cuneus \\
\hline 9 & 479 & 6.68 & 50 & -70 & 0 & R MTG/STG/ITG/IPL/SMG/AG/MOG/IOG \\
\hline 10 & 153 & 5.62 & 62 & -46 & -8 & R MTG/STG \\
\hline 11 & 164 & 5.97 & -38 & 50 & 4 & L IFG/TrilFG/OrbIFG/MFG/SFG \\
\hline 12 & 100 & 6.68 & -10 & -84 & 4 & LSOG/cuneus \\
\hline 13 & 614 & 13.03 & -2 & -56 & 26 & LPCu/cuneus \\
\hline 14 & 71 & 7.03 & 50 & -14 & 10 & R PreCG/PoCG/RO/SMG/STG \\
\hline 15 & 1070 & 16.21 & 0 & 14 & 48 & SMA/MedFG/SupMFG \\
\hline 16 & 468 & 10.21 & 34 & -56 & 44 & $\mathrm{R} I \mathrm{PL} / \mathrm{SPL} / \mathrm{PCU} / \mathrm{SMG} / \mathrm{AG} / \mathrm{SOG}$ \\
\hline 17 & 60 & 4.91 & -10 & -66 & 52 & L SPL/PCu \\
\hline 18 & 36 & 5.97 & -28 & 28 & 48 & LMFG/SFG \\
\hline 19 & 86 & 5.97 & -30 & -2 & 56 & L MFG/SFG/PreCG \\
\hline
\end{tabular}

$\mathrm{L}$, left; $\mathrm{R}$, right; $\mathrm{SFG}$, superior frontal gyrus; $\mathrm{MFG}$, middle frontal gyrus; IFG, inferior frontal gyrus; OrbIFG, orbital inferior frontal gyrus; TrilFG, triangular inferior frontal gyrus; OperIFG, opercular inferior frontal gyrus; SupMFG, superior medial frontal gyrus; MedFG, medial frontal gyrus; OrbMFG, orbital medial frontal gyrus; STG, superior temporal gyrus; MTG, middle temporal gyrus; ITG, inferior temporal gyrus; RO, Rolandic operculum; SPL, superior parietal lobule; IPL, inferior parietal lobule; SMG, supramarginal gyrus; AG, angular gyrus; PreCG, precentral gyrus; PoCG, postcentral gyrus; PCu, precuneus; SMA, supplementary motor area; SOG, superior occipital gyrus; MOG, middle occipital gyrus; IOG, inferior occipital gyrus 
Table 4 Coordinates of bipolar disorder-associated surface regions identified from meta-analysis

\begin{tabular}{|c|c|c|c|c|c|c|}
\hline \multirow[t]{2}{*}{ Cluster ID } & \multirow[t]{2}{*}{ Cluster size } & \multirow[t]{2}{*}{ Peak T } & \multicolumn{3}{|c|}{ Peak coordinates } & \multirow[t]{2}{*}{ Brain regions } \\
\hline & & & $x$ & y & $\mathbf{z}$ & \\
\hline 1 & 55 & 7.29 & -40 & 18 & -20 & L IFG/OrbIFG/STP \\
\hline 2 & 60 & 7.29 & 40 & -78 & -4 & RMOG/IOG/ITG \\
\hline 3 & 37 & 7.29 & -58 & -14 & 6 & LSTG \\
\hline 4 & 38 & 5.84 & 30 & 40 & 24 & R MFG/SFG \\
\hline 5 & 87 & 8.74 & 46 & 4 & 30 & R IFG/OperIFG/MFG/PreCG \\
\hline 6 & 54 & 6.57 & 32 & -54 & 40 & RIPL/SPL/SMG/AG \\
\hline 7 & 72 & 8.02 & -30 & -58 & 44 & L IPL/SPL \\
\hline 8 & 205 & 8.02 & -4 & 8 & 52 & L MedFG/SFG/SMA \\
\hline
\end{tabular}

L, left; R, right; SFG, superior frontal gyrus; MFG, middle frontal gyrus; IFG, inferior frontal gyrus; OrbIFG, orbital inferior frontal gyrus; OperlFG, opercular inferior frontal gyrus; MedFG, medial frontal gyrus; STG, superior temporal gyrus; ITG, inferior temporal gyrus; STP, superior temporal pole; SPL, superior parietal lobule; IPL, inferior parietal lobule; SMG, supramarginal gyrus; AG, angular gyrus; PreCG, precentral gyrus; SMA, supplementary motor area; MOG, middle occipital gyrus; IOG, inferior occipital gyrus

Table 5 Coordinates of compulsive disorder -associated surface regions identified from meta-analysis

\begin{tabular}{|c|c|c|c|c|c|c|}
\hline \multirow[t]{2}{*}{ Cluster ID } & \multirow[t]{2}{*}{ Cluster size } & \multirow[t]{2}{*}{ Peak T } & \multicolumn{3}{|c|}{ Peak coordinates } & \multirow[t]{2}{*}{ Brain regions } \\
\hline & & & $\mathbf{x}$ & y & $\mathbf{z}$ & \\
\hline 1 & 153 & 7.62 & -54 & -2 & -16 & L MTG/STG/ITG/STP \\
\hline 2 & 67 & 5.86 & 46 & -76 & -8 & R MOG/IOG/MTG/ITG \\
\hline 3 & 31 & 5.87 & -38 & 32 & -10 & LIFG/OrbIFG/MFG \\
\hline 4 & 846 & 9.38 & -64 & -34 & 4 & L MTG/STG/ITG/IPL/SMG/MOG/IOG \\
\hline 5 & 38 & 6.75 & 38 & 24 & -8 & RIFG/OrbIFG \\
\hline 6 & 39 & 5.87 & -34 & 26 & -8 & L IFG/OrbIFG/TrilFG \\
\hline 7 & 33 & 5.87 & 22 & -92 & -6 & RIOG/MOG/cuneus \\
\hline 8 & 1544 & 10.26 & -54 & 10 & 18 & L IFG/TrilFG/OperIFG/MFG/PreCG/PoCG/RO \\
\hline 9 & 32 & 5.87 & -36 & -86 & -6 & LMOG/IOG \\
\hline 10 & 285 & 7.62 & 62 & -34 & 0 & R STG/MTG \\
\hline 11 & 149 & 6.75 & -62 & -14 & 6 & L STG/PreCG/PoCG/RO \\
\hline 12 & 54 & 6.75 & 60 & -4 & 16 & R PreCG/PoCG/RO \\
\hline 13 & 34 & 4.99 & 50 & 6 & 20 & R IFG/OperIFG/TrilFG/PreCG \\
\hline 14 & 79 & 6.75 & 54 & 28 & 22 & R MFG/IFG/TrilFG \\
\hline 15 & 66 & 5.87 & 48 & -4 & 44 & R MFG/PreCG/PoCG \\
\hline 16 & 320 & 7.62 & -2 & 14 & 52 & L MedFG/SupMFG/SFG/SMA \\
\hline 17 & 71 & 6.75 & -30 & -72 & 44 & LSPL/IPL/PCu/MOG/AG \\
\hline
\end{tabular}

L, left; R, right; SFG, superior frontal gyrus; MFG, middle frontal gyrus; IFG, inferior frontal gyrus; OrbIFG, orbital inferior frontal gyrus; TrilFG, triangular inferior frontal gyrus; OperIFG, opercular inferior frontal gyrus; SupMFG, superior medial frontal gyrus; MedFG, medial frontal gyrus; STG, superior temporal gyrus; MTG, middle temporal gyrus; ITG, inferior temporal gyrus; STP, superior temporal pole; SPL, superior parietal lobule; IPL, inferior parietal lobule; SMG, supramarginal gyrus; AG, angular gyrus; PreCG, precentral gyrus; PoCG, postcentral gyrus; PCu, precuneus; SMA, supplementary motor area; RO, Rolandic operculum; MOG, middle occipital gyrus; IOG, inferior occipital gyrus

supplementary motor area (SMA), as well as the right supramarginal gyrus (SMG) and inferior occipital gyrus (IOG) (Table 6, Fig. 1F).

\section{G. PTSD}

106 studies (extracted from Neurosynth) were included in the data analysis (a complete list of the 106 studies can be found in Additional file 1: Table S7). Fourteen clusters on the brain surface were identified from the uniformity test map of the meta-analysis (Table 7).

These brain regions were the bilateral superior frontal gyrus (SFG), middle frontal gyrus (MFG), inferior frontal gyrus (IFG), orbital inferior frontal gyrus (OrbIFG), triangular inferior frontal gyrus (TriIFG), opercular inferior frontal gyrus (OperIFG), superior parietal lobule (SPL), inferior parietal lobule (IPL), supramarginal gyrus 
Table 6 Coordinates of major depression-associated surface regions identified from meta-analysis

\begin{tabular}{|c|c|c|c|c|c|c|}
\hline \multirow[t]{2}{*}{ Cluster ID } & \multirow[t]{2}{*}{ Cluster size } & \multirow[t]{2}{*}{ Peak T } & \multicolumn{3}{|c|}{ Peak coordinates } & \multirow[t]{2}{*}{ Brain regions } \\
\hline & & & $\mathrm{x}$ & y & z & \\
\hline 1 & 441 & 7.76 & 42 & -78 & -6 & RIOG/MOG/STG/ITG/MTG \\
\hline 2 & 48 & 4.80 & 56 & -6 & -22 & R MTG/ITG/STG \\
\hline 3 & 86 & 7.17 & -60 & -14 & -14 & L MTG/ITG \\
\hline 4 & 858 & 9.54 & -46 & 26 & -10 & L IFG/OrbIFG/TrilFG/OperlFG/MFG/PreCG/STG \\
\hline 5 & 51 & 7.76 & 34 & 22 & -10 & R IFG/OrbIFG \\
\hline 6 & 351 & 7.17 & -2 & 58 & 20 & L MedFG/SupMFG/OrbMFG/SFG \\
\hline 7 & 271 & 8.36 & -46 & -66 & 0 & L MOG/MTG/ITG \\
\hline 8 & 769 & 8.95 & 36 & 28 & 2 & R IFG/OrbIFG/TrilFG/OperlFG/MFG/PreCG \\
\hline 9 & 78 & 5.99 & -56 & -42 & 2 & L MTG/STG \\
\hline 10 & 141 & 6.58 & 54 & -32 & 6 & R MTG/STG \\
\hline 11 & 236 & 7.17 & 50 & -58 & 24 & R STG/MTG/IPL/SMG/AG \\
\hline 12 & 227 & 6.58 & 2 & -54 & 24 & RPCu/PoCG \\
\hline 13 & 90 & 5.99 & -48 & -66 & 28 & L MTG/STG/IPL/AG \\
\hline 14 & 34 & 4.80 & 50 & -60 & 38 & RIPL/SMG/AG \\
\hline 15 & 349 & 8.36 & -2 & 10 & 52 & L MedFG/SMA \\
\hline 16 & 190 & 5.99 & -40 & -42 & 48 & L IPL/SPL/PCu/PoCG \\
\hline 17 & 59 & 4.80 & -46 & -30 & 52 & LIPL/PoCG \\
\hline 18 & 53 & 5.39 & -26 & 0 & 56 & L MFG/SFG/PreCG \\
\hline
\end{tabular}

L, left; $R$, right; $S F G$, superior frontal gyrus; MFG, middle frontal gyrus; IFG, inferior frontal gyrus; OrbIFG, orbital inferior frontal gyrus; TrilFG, triangular inferior frontal gyrus; OperIFG, opercular inferior frontal gyrus; SupMFG, superior medial frontal gyrus; MedFG, medial frontal gyrus; OrbMFG, orbital medial frontal gyrus; STG, superior temporal gyrus; MTG, middle temporal gyrus; ITG, inferior temporal gyrus; SPL, superior parietal lobule; IPL, inferior parietal lobule; SMG, supramarginal gyrus; AG, angular gyrus; PreCG, precentral gyrus; PoCG, postcentral gyrus; PCu, precuneus; SMA, supplementary motor area; MOG, middle occipital gyrus; IOG, inferior occipital gyrus

Table 7 Coordinates of PTSD-associated surface regions identified from meta-analysis

\begin{tabular}{|c|c|c|c|c|c|c|}
\hline \multirow[t]{2}{*}{ Cluster ID } & \multirow[t]{2}{*}{ Cluster size } & \multirow[t]{2}{*}{ Peak T } & \multicolumn{3}{|c|}{ Peak coordinates } & \multirow[t]{2}{*}{ Brain regions } \\
\hline & & & $\mathrm{x}$ & y & z & \\
\hline 1 & 273 & 8.26 & 50 & 22 & -10 & R IFG/OrbIFG/TrilFG/OperlFG \\
\hline 2 & 169 & 8.26 & -46 & 20 & -4 & LIFG/OrbIFG/TrilFG \\
\hline 3 & 57 & 6.20 & -44 & 40 & -6 & L MFG/OrblFG \\
\hline 4 & 95 & 6.20 & -42 & 28 & 14 & LIFG/TrilFG/MFG \\
\hline 5 & 47 & 5.18 & -48 & 8 & 32 & L IFG/TrilFG/OperIFG/PreCG \\
\hline 6 & 54 & 5.18 & -36 & 36 & 28 & L MFG/SFG \\
\hline 7 & 40 & 7.23 & -56 & -34 & 36 & LIPL/SMG/PoCG \\
\hline 8 & 54 & 7.23 & -34 & 10 & 40 & L MFG/PreCG \\
\hline 9 & 345 & 8.26 & -2 & 20 & 42 & L SMA/MedFG/SupMFG \\
\hline 10 & 184 & 8.26 & -40 & -54 & 44 & LIPL/SPL/PCu/AG \\
\hline 11 & 36 & 5.18 & 34 & -70 & 44 & $\mathrm{RSPL} / \mathrm{PCu} / \mathrm{AG}$ \\
\hline 12 & 98 & 7.23 & 44 & -42 & 48 & R IPL/SMG/PoCG \\
\hline 13 & 60 & 7.23 & 34 & 10 & 56 & R MFG/SFG \\
\hline 14 & 34 & 4.15 & -24 & 2 & 62 & LMFG/SFG \\
\hline
\end{tabular}

L, left; $R$, right; $S F G$, superior frontal gyrus; $M F G$, middle frontal gyrus; IFG, inferior frontal gyrus; OrblFG, orbital inferior frontal gyrus; TrilFG, triangular inferior frontal gyrus; OperlFG, opercular inferior frontal gyrus; SupMFG, superior medial frontal gyrus; MedFG, medial frontal gyrus; SPL, superior parietal lobule; IPL, inferior parietal lobule; SMG, supramarginal gyrus; AG, angular gyrus; PreCG, precentral gyrus; PoCG, postcentral gyrus; $\mathrm{PCu}$, precuneus; SMA, supplementary motor area 
(SMG), angular gyrus (AG), postcentral gyrus (PoCG) and precuneus $(\mathrm{PCu})$, as well as the left medial frontal gyrus (MedFG), superior medial frontal gyrus (SupMFG), precentral gyrus (PreCG) and supplementary motor area (SMA) (Table 7, Fig. 1G).

\section{H. Schizophrenia}

715 studies (extracted from Neurosynth) were included in the data analysis (a complete list of the 715 studies can be found in Additional file 1: Table S8). Eight clusters on the brain surface were identified from the uniformity test map of the meta-analysis (Table 8).

These brain regions were the bilateral middle frontal gyrus (MFG), inferior frontal gyrus (IFG), triangular inferior frontal gyrus (TriIFG), opercular inferior frontal gyrus (OperIFG), precentral gyrus (PreCG), as well as the left superior frontal gyrus (SFG), orbital inferior frontal gyrus (OrbIFG), medial frontal gyrus (MedFG), superior medial frontal gyrus (SupMFG), orbital medial frontal gyrus (OrbMFG), middle temporal gyrus (MTG), inferior temporal gyrus (ITG), supplementary motor area (SMA) and middle occipital gyrus (MOG) (Table 8, Fig. 1H).

\section{Neuroimaging-based scalp stimulation locations}

To facilitate clinical application, we further refined/simplified the results and proposed neuroimaging-based target protocols for each disorder respectively. We accomplished this by identifying the eight or nine surface regions with peak coordinates of each cluster for each disorder respectively, based on the findings from the meta-analysis. We applied two methods, the 10-20 EEG system and the international standard acupoints, to identify the potential scalp stimulation locations. To help the readers understand the specific brain functions of identified areas, we also summarized the brain functions of each identified brain region associated with a corresponding mental disorder. We named the potential targets in the order of left, top, right, front, and back view of the head. Detailed descriptions of potential targets for each disorder based on 10-20 EEG system coordinates and acupuncture points can be found in Fig. 2, and Tables 9, 10, 11, 12, 13, 14, 15 and 16, respectively.

\section{A. ADHD}

We proposed nine potential targets for treating ADHD (named ADHD-1 to ADHD-9). These targets were located mainly in the frontal gyrus, precentral gyrus, precuneus, parietal lobe, supramarginal gyrus, and angular gyrus (Table 9, Fig. 2A).

\section{B. Anxiety disorder}

We proposed nine potential targets for treating anxiety disorder (named ANX-1 to ANX-9). These targets were located mainly in the frontal gyrus, supplementary motor area, temporal gyrus, and occipital gyrus (Table 10, Fig. 2B).

\section{Autism spectrum disorder}

We proposed nine potential targets for treating autism spectrum disorder (named AUT-1 to AUT-9). These targets were located mainly in the frontal gyrus, supplementary motor area, precentral gyrus, precuneus, temporal gyrus, parietal lobe, supramarginal gyrus, and angular gyrus (Table 11, Fig. 2C).

\section{Bipolar disorder}

We proposed eight potential targets for treating bipolar disorder (named BD-1 to BD-8). These targets were located mainly in the frontal gyrus, supplementary motor area, precentral gyrus, precuneus, temporal gyrus,

Table 8 Coordinates of schizophrenia-associated surface regions identified from meta-analysis

\begin{tabular}{|c|c|c|c|c|c|c|}
\hline \multirow[t]{2}{*}{ Cluster ID } & \multirow[t]{2}{*}{ Cluster size } & \multirow[t]{2}{*}{ Peak T } & \multicolumn{3}{|c|}{ Peak coordinates } & \multirow[t]{2}{*}{ Brain regions } \\
\hline & & & $\mathbf{x}$ & $y$ & z & \\
\hline 1 & 36 & 4.62 & -6 & 38 & -14 & L MedFG/OrbMFG \\
\hline 2 & 39 & 6.83 & -48 & -70 & -4 & L MOG/ITG/MTG \\
\hline 3 & 104 & 7.94 & -34 & 40 & 2 & L TrilFG/OrbIFG/MFG \\
\hline 4 & 35 & 5.73 & -16 & 58 & 8 & L MedFG/SupMFG/SFG \\
\hline 5 & 288 & 10.15 & 46 & 24 & 24 & RTrilFG/OperIFG/IFG/MFG \\
\hline 6 & 47 & 6.83 & 42 & 6 & 28 & R OperlFG/IFG/PreCG \\
\hline 7 & 105 & 6.83 & -46 & 8 & 30 & L IFG/TrilFG/OperlFG/MFG/PreCG \\
\hline 8 & 163 & 10.15 & -2 & 22 & 40 & L SupMFG/MedFG/SMA \\
\hline
\end{tabular}

L, left; $R$, right; $S F G$, superior frontal gyrus; MFG, middle frontal gyrus; IFG, inferior frontal gyrus; OrbIFG, orbital inferior frontal gyrus; TrilFG, triangular inferior frontal gyrus; OperlFG, opercular inferior frontal gyrus; SupMFG, superior medial frontal gyrus; MedFG, medial frontal gyrus; OrbMFG, orbital medial frontal gyrus; MTG, middle temporal gyrus; ITG, inferior temporal gyrus; PreCG, precentral gyrus; SMA, supplementary motor area; MOG, middle occipital gyrus 


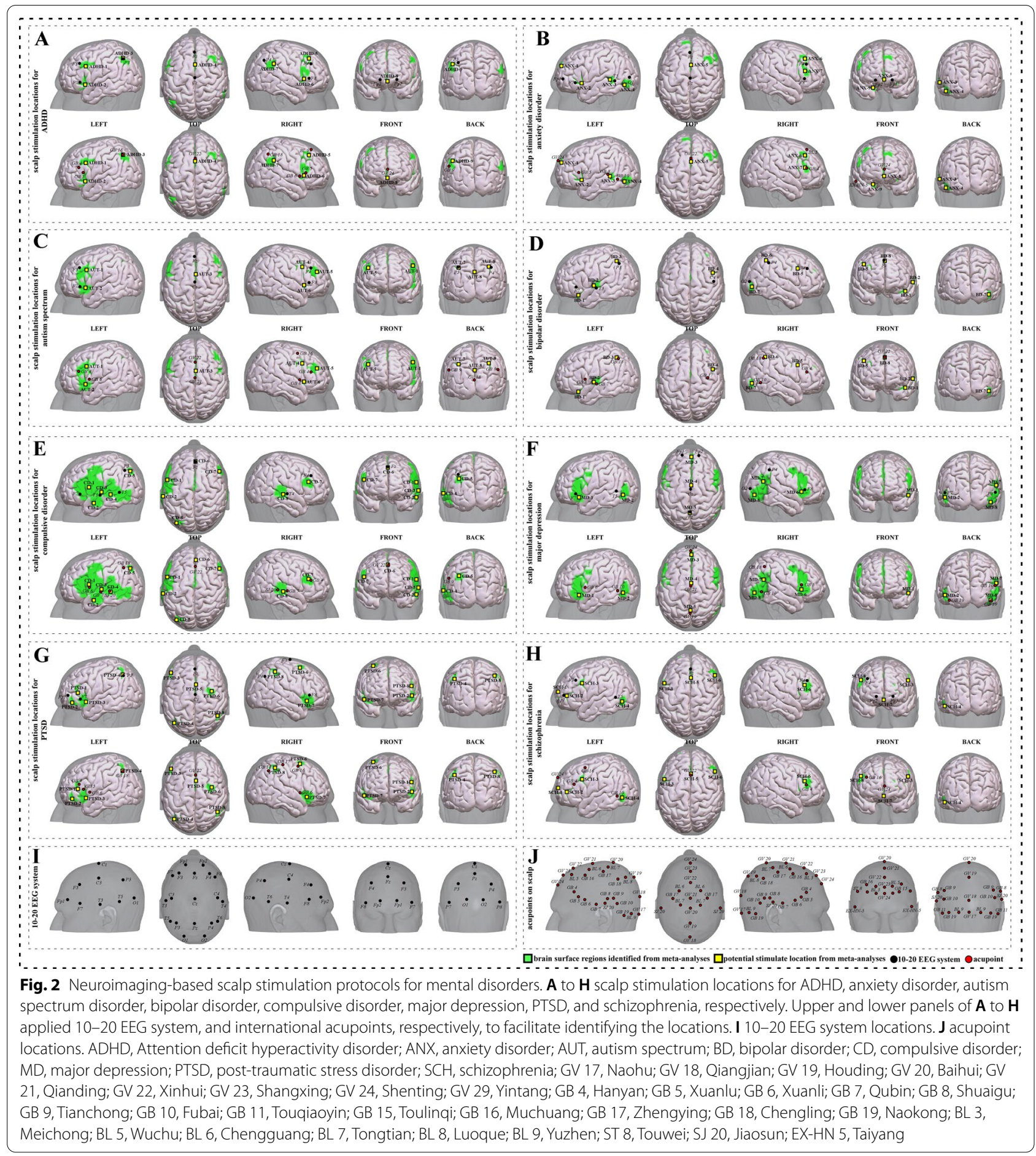

parietal lobe, supramarginal gyrus, angular gyrus, and occipital gyrus (Table 12, Fig. 2D).

\section{E. Compulsive disorder}

We proposed eight potential targets for treating compulsive disorder (named CD-1 to CD-8). These targets were located mainly in the frontal gyrus, supplementary motor area, precentral and postcentral gyrus, temporal gyrus, parietal lobe, supramarginal gyrus, angular gyrus, and occipital gyrus (Table 13, Fig. 2E). 


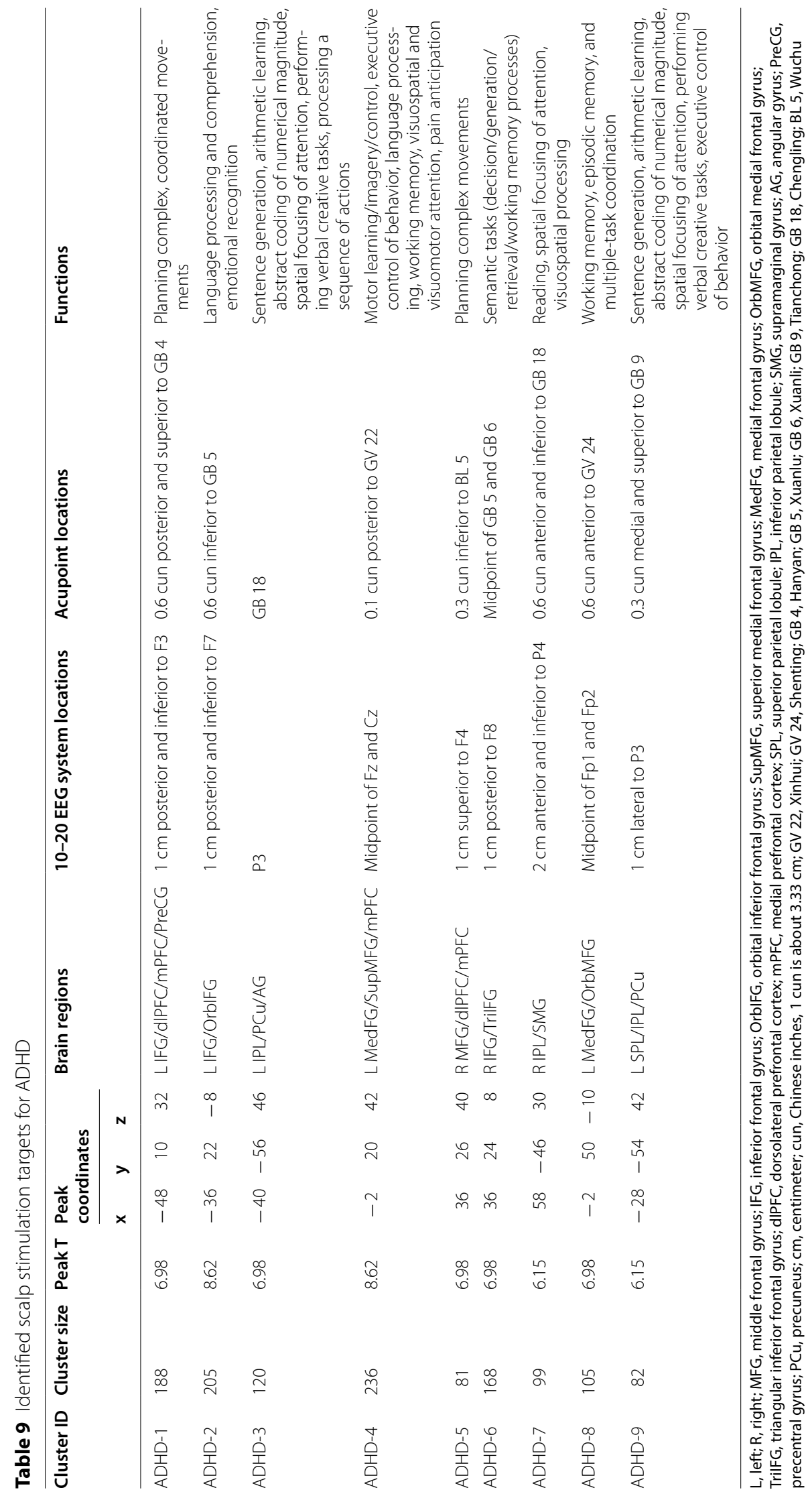




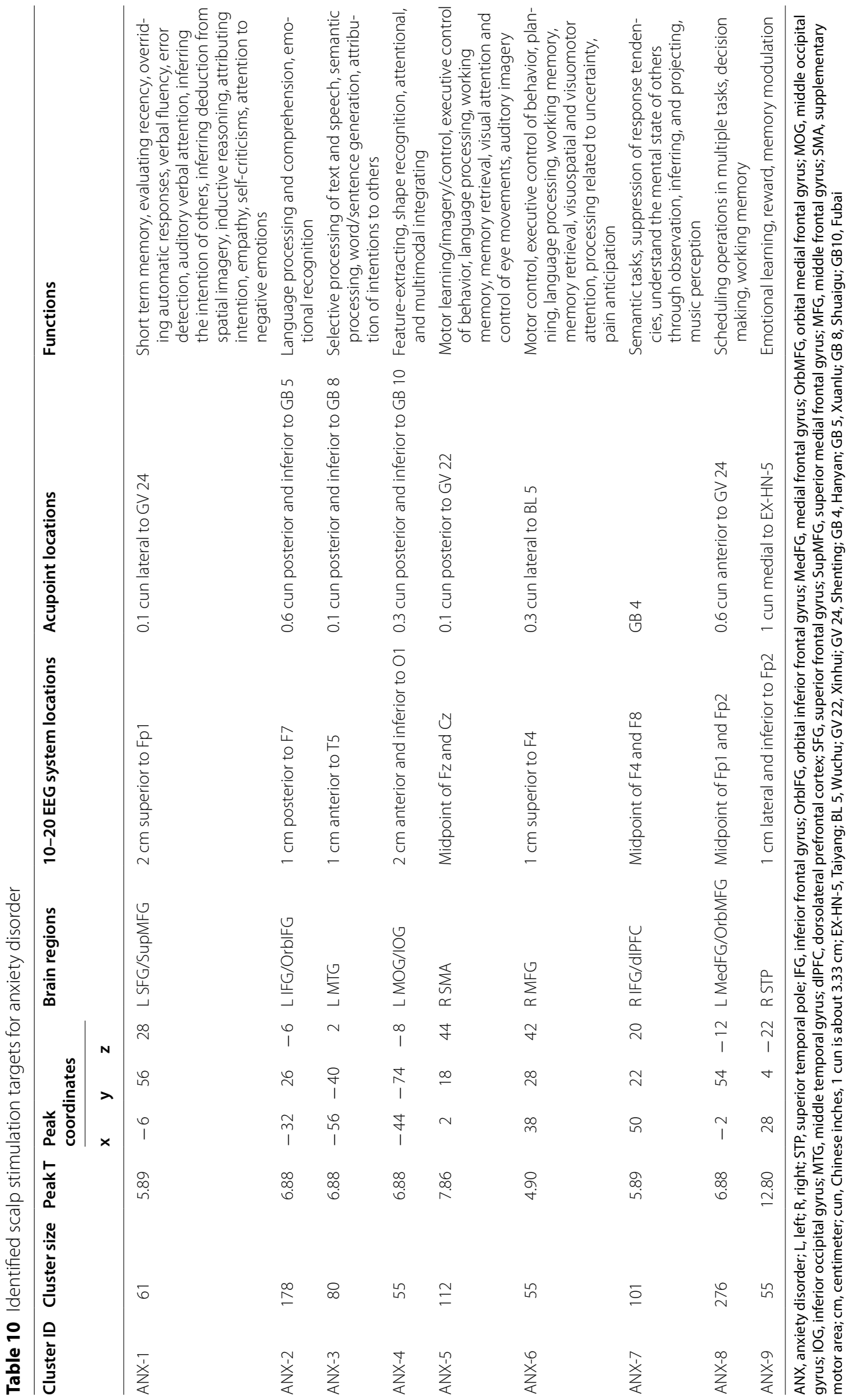




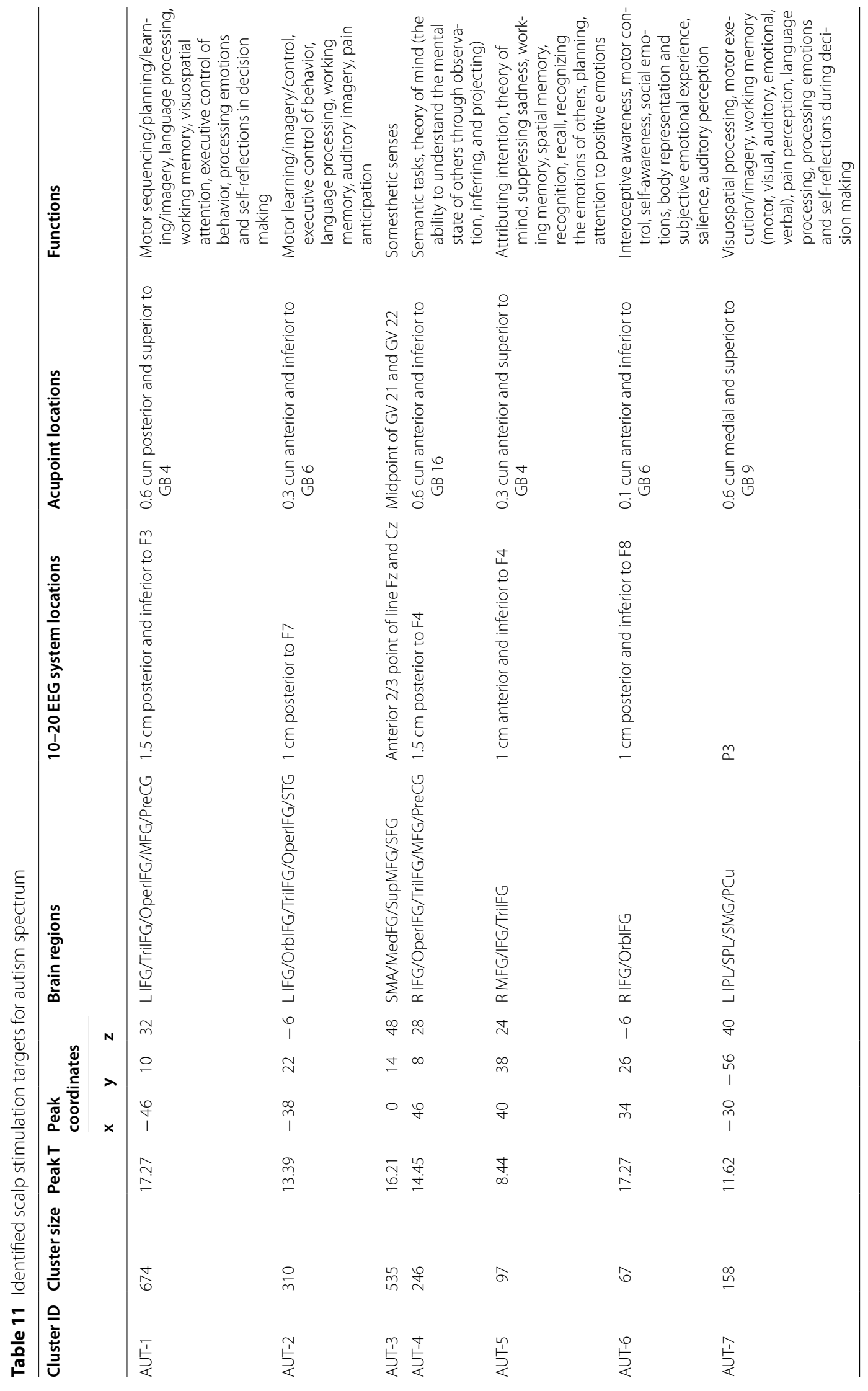




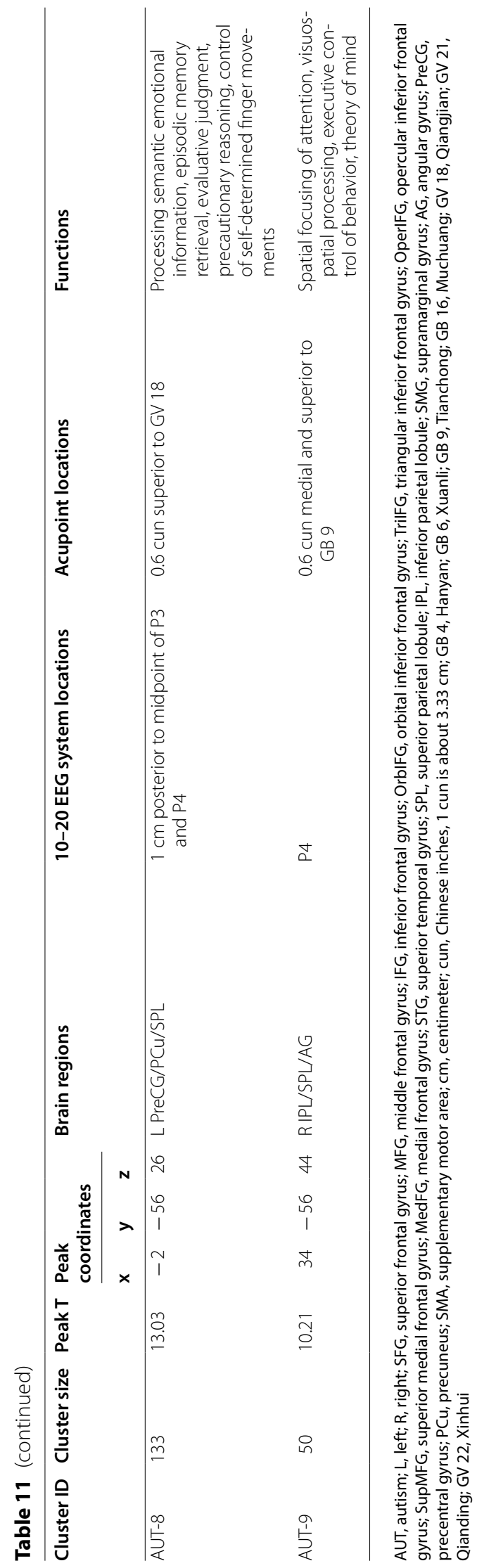




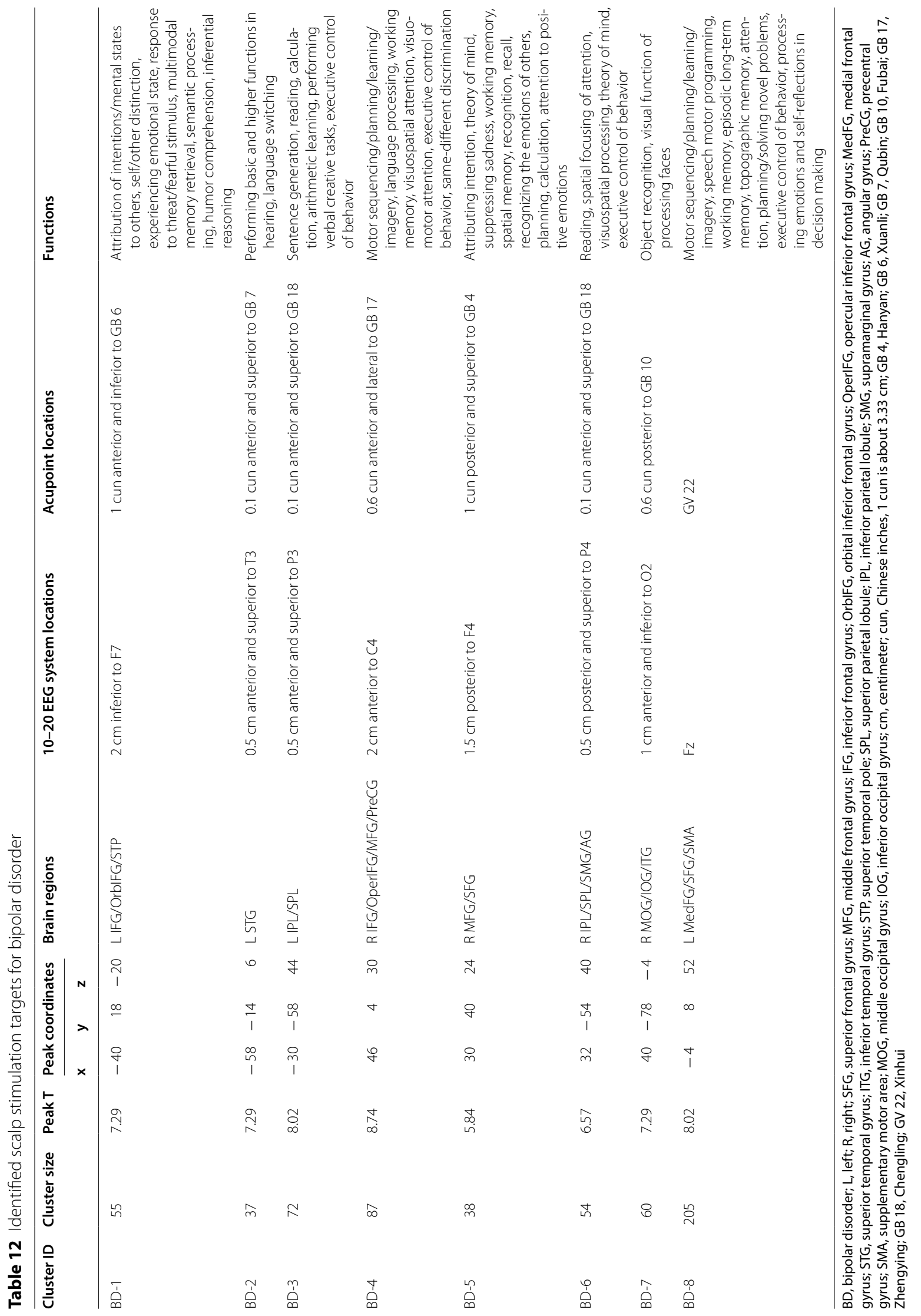




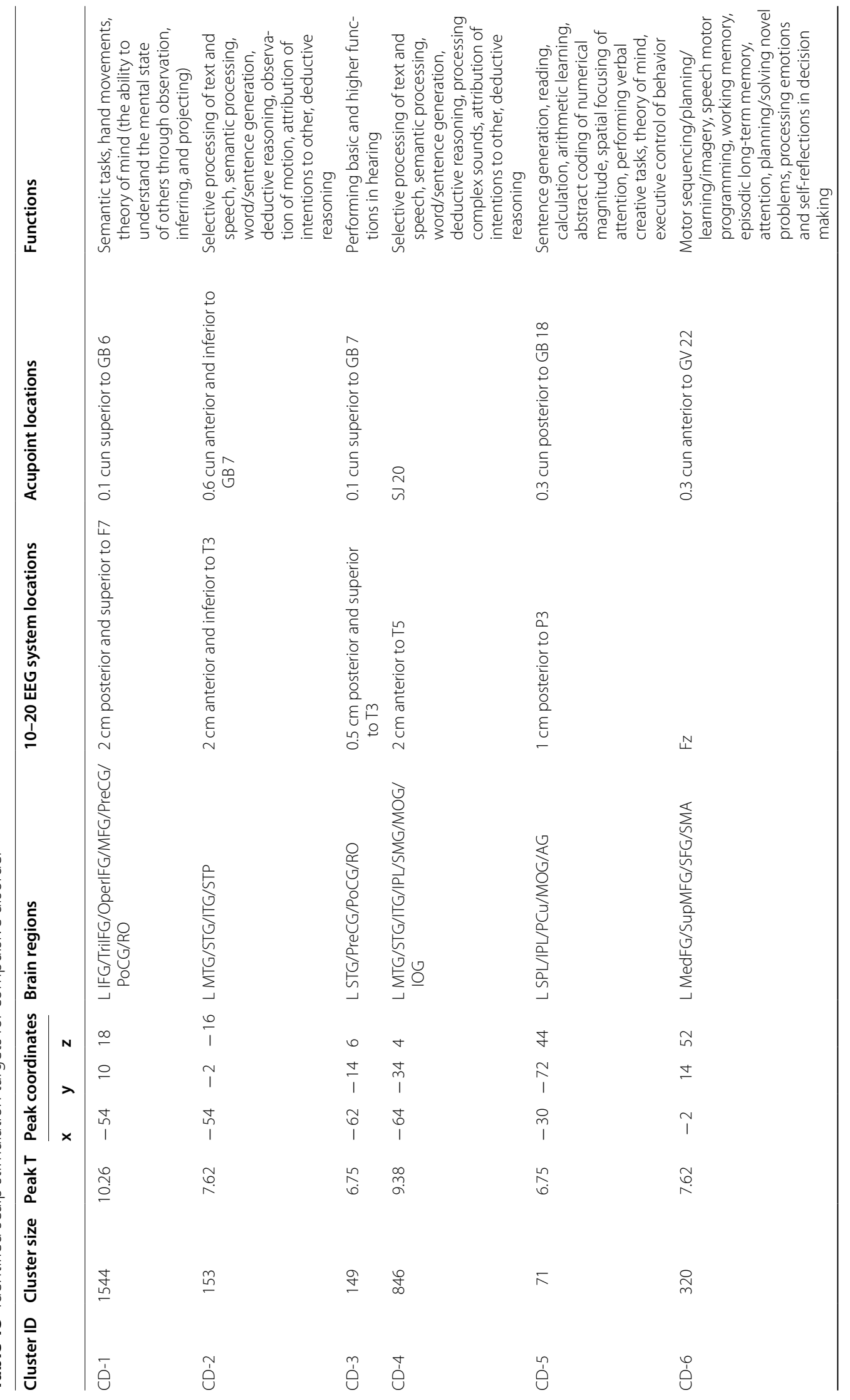




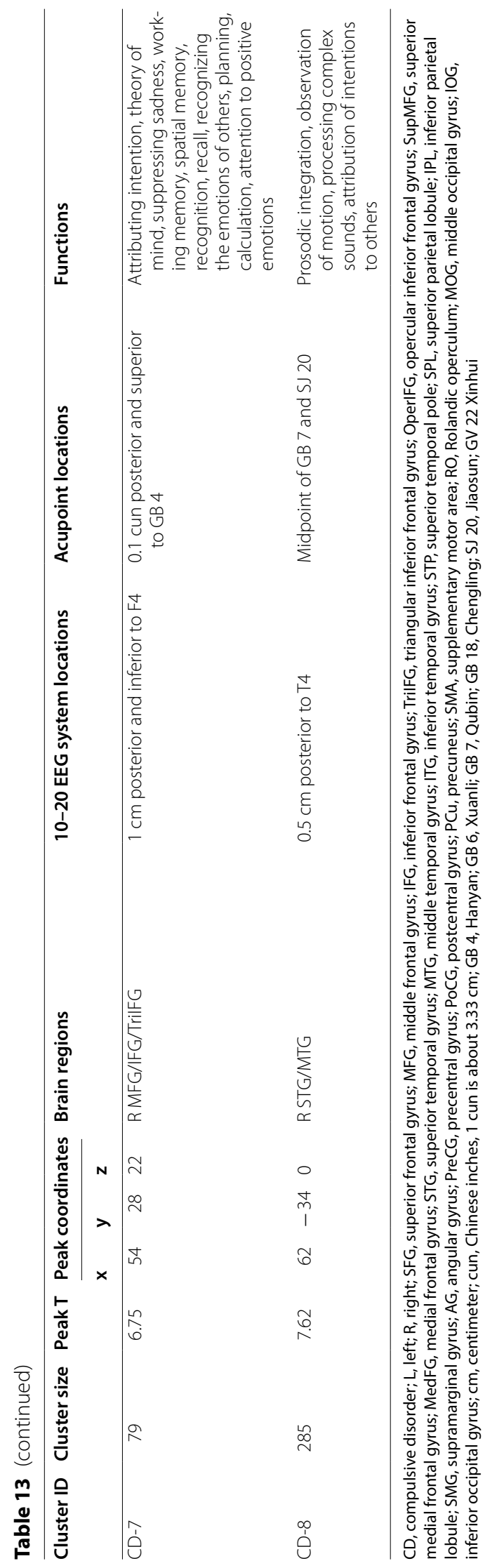




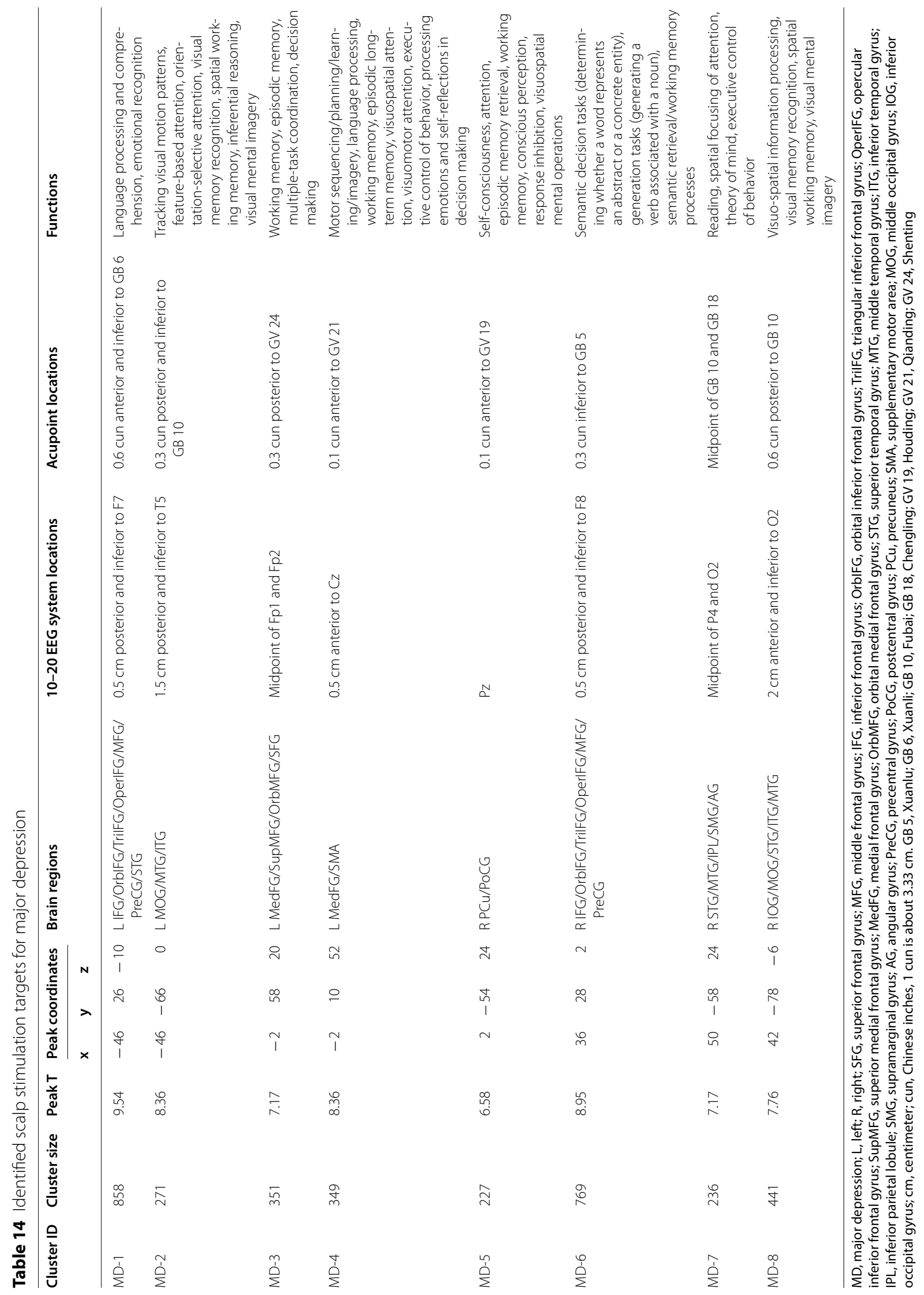




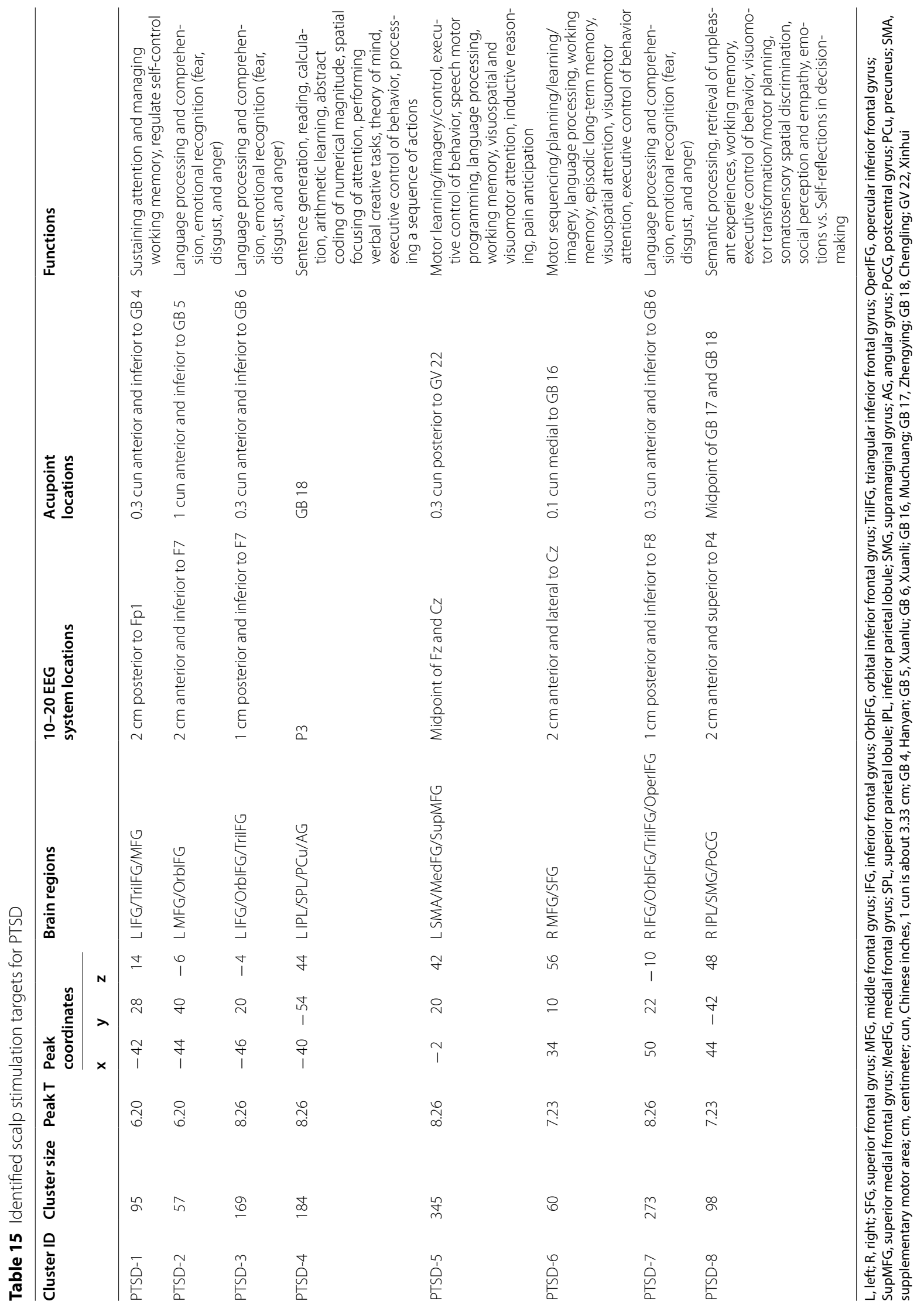




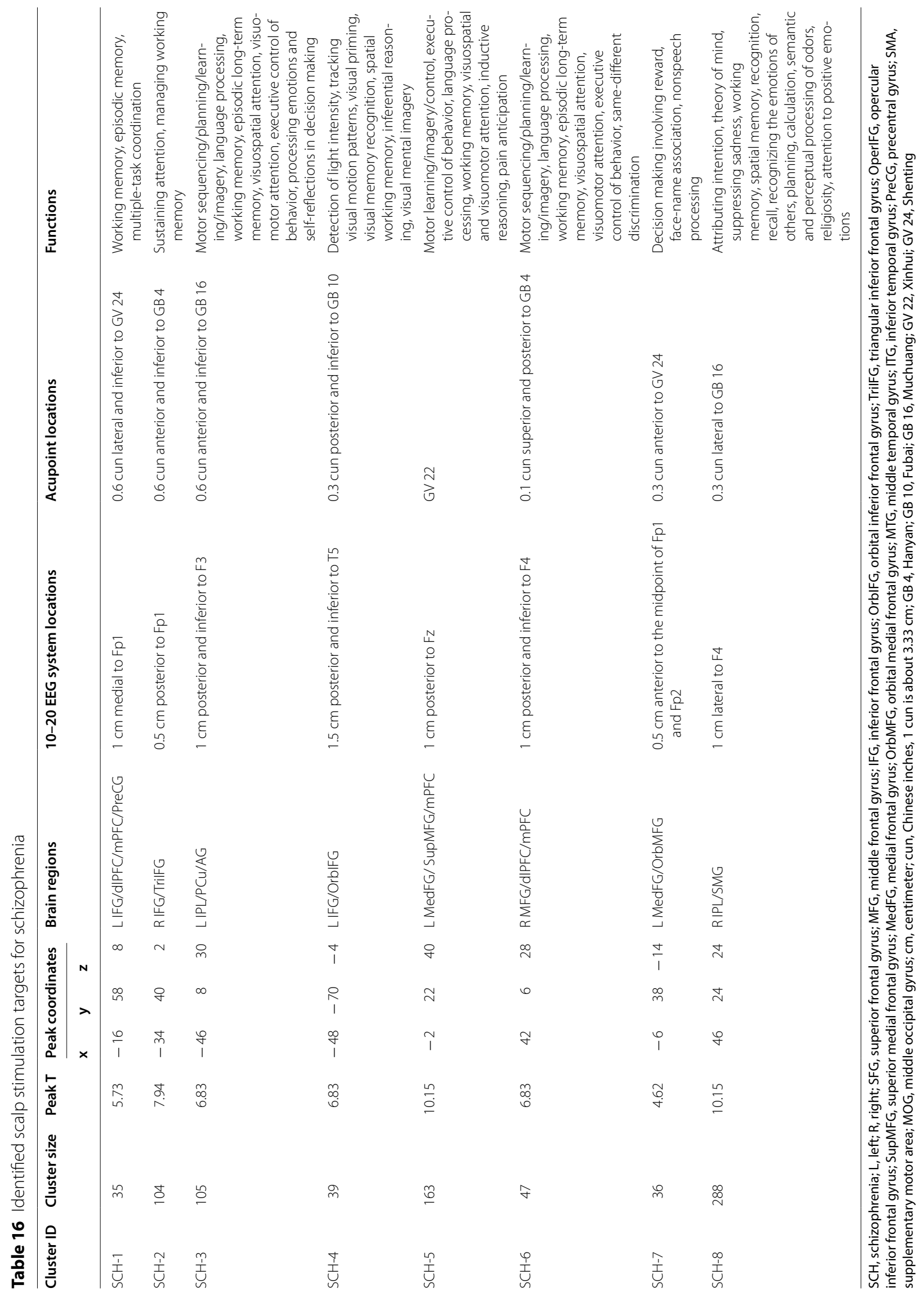




\section{F. Major depression}

We proposed eight potential targets for treating major depression (named MD-1 to MD-8). These targets were located mainly in the frontal gyrus, supplementary motor area, precentral and postcentral gyrus, precuneus, temporal gyrus, parietal lobe, supramarginal gyrus, angular gyrus, and occipital gyrus (Table 14, Fig. 2F).

\section{G. PTSD}

We proposed eight potential targets for treating PTSD (named PTSD-1 to PTSD-8). These targets were located mainly in the frontal gyrus, supplementary motor area, postcentral gyrus, precuneus, temporal gyrus, parietal lobe, supramarginal gyrus, and angular gyrus (Table 15, Fig. 2G).

\section{H. Schizophrenia}

We proposed eight potential targets for treating schizophrenia (named SCH-1 to SCH-8). These targets were located mainly in the frontal gyrus, supplementary motor area, precentral gyrus, precuneus, temporal gyrus, and occipital gyrus (Table 16, Fig. 2H).

\section{Overlapped surface regions among mental disorders}

We also explored overlap regions across different mental disorders (Table 17 and Fig. 3). We found that: a) ADHD, PTSD, anxiety disorder, autism spectrum disorder, compulsive disorder, major depression, and schizophrenia show an overlap on the medial frontal gyrus (MedFG)/ SMA (Fig. 3A); b) the right dorsal lateral prefrontal cortex (dlPFC) is involved in anxiety disorder, autism spectrum disorder, bipolar disorder, major depression, and schizophrenia (Fig. 3B); c) the left IFG/lateral orbital prefrontal cortex (OrbPFC) contributed to ADHD, PTSD, anxiety disorder, autism spectrum disorder, and major depression (Fig. 3C); d) anxiety disorder, compulsive disorder, major depression, and schizophrenia display an overlap on the left MTG/STG/ITG//IOG/MOG (Fig. 3D); e) the orbital medial frontal gyrus (OrbMFG)/ ventral medial frontal gyrus (VenMFG) are associated with ADHD, anxiety disorder, major depression, and schizophrenia (Fig. 3E); f) ADHD, autism spectrum disorder, and bipolar disorder are allying with the left IPL/ SPL/SMG/AG (Fig. 3F); g) autism spectrum disorder and major depression have an overlap region in the $\mathrm{PCu}$ (Fig. 3G); h) autism spectrum disorder and bipolar disorder overlap in the right IPL/SPL/SMG/AG (Fig. 3H); and i) the right ITG/MTG/STG/IOG/MOG areas are involved in major depression and bipolar disorder (Fig. 3I).

\section{Discussion}

In this study, we investigated potential targets for scalp stimulation (scalp acupuncture/transcranial stimulation) in the treatment of eight common mental disorders using a meta-analysis method that incorporates text-mining, meta-analysis and machine-learning techniques. To further facilitate its clinical application, we selected eight to nine potential targets (peak coordinates mapped onto brain surface using MRIcroGL and SurfIce) for each disorder based on the significance of each brain region derived from the meta-analysis. Finally, we used the 10-20 EEG system and existing acupoints to aid in locating corresponding regions on the scalp for these targets.

Table 17 Overlap surface regions among different mental disorders

\begin{tabular}{|c|c|c|c|c|c|}
\hline \multirow[t]{2}{*}{ Cluster ID } & \multicolumn{3}{|c|}{$\begin{array}{l}\text { Peak } \\
\text { coordinates }\end{array}$} & \multirow[t]{2}{*}{ Overlap brain regions } & \multirow[t]{2}{*}{ Overlap disorders } \\
\hline & $\mathrm{x}$ & $\mathbf{y}$ & z & & \\
\hline A & 2 & 22 & 42 & R SMA/MedFG & $\begin{array}{l}7 \text { disorders: ADHD, PTSD, anxiety disorder, autism spectrum disorder, compulsive disorder, } \\
\text { major depression, schizophrenia }\end{array}$ \\
\hline B & 42 & 6 & 28 & R dIPFC & $\begin{array}{l}5 \text { disorders: anxiety disorder, autism spectrum, bipolar disorder, major depression, schizo- } \\
\text { phrenia }\end{array}$ \\
\hline C & -34 & 26 & -4 & L IFG/lateral OrbPFC & 5 disorders: ADHD, PTSD, anxiety disorder, autism spectrum disorder, major depression \\
\hline D & -46 & -68 & -4 & L MTG/STG/ITG//IOG/MOG & 4 disorders: anxiety disorder, compulsive disorder, major depression, schizophrenia \\
\hline E & -2 & 40 & -12 & L OrbMFG/NenMedFG & 4 disorders: ADHD, anxiety disorder, major depression, schizophrenia \\
\hline $\mathrm{F}$ & -30 & -52 & 42 & LIPL/SPL/SMG/AG & 3 disorders: ADHD, autism spectrum disorder, bipolar disorder \\
\hline G & 2 & -54 & 20 & $\mathrm{RPCu}$ & 2 disorders: autism spectrum disorder, major depression \\
\hline $\mathrm{H}$ & 34 & -52 & 40 & $\mathrm{RIPL/SPL/SMG/AG}$ & 2 disorders: autism spectrum disorder, bipolar disorder \\
\hline । & 42 & -78 & -8 & R ITG/MTG/STG/IOG/MOG & 2 disorders: major depression, bipolar disorder \\
\hline
\end{tabular}

L, left; R, right; IFG, inferior frontal gyrus; MedFG, medial frontal gyrus; OrbMFG, orbital medial frontal gyrus; VenMFG ventral medial frontal gyrus; dIPFC, dorsal lateral prefrontal cortex; OrbPFC, orbital prefrontal cortex; MTG, middle temporal gyrus; ITG, inferior temporal gyrus; STG, superior temporal gyrus; IPL, inferior parietal lobe; SPL, superior parietal lobe; SMA, supplementary motor area; SMG, supramarginal gyrus; AG, angular gyrus; PCu, precuneus; MOG, middle occipital gyrus; IOG, inferior occipital gyrus 


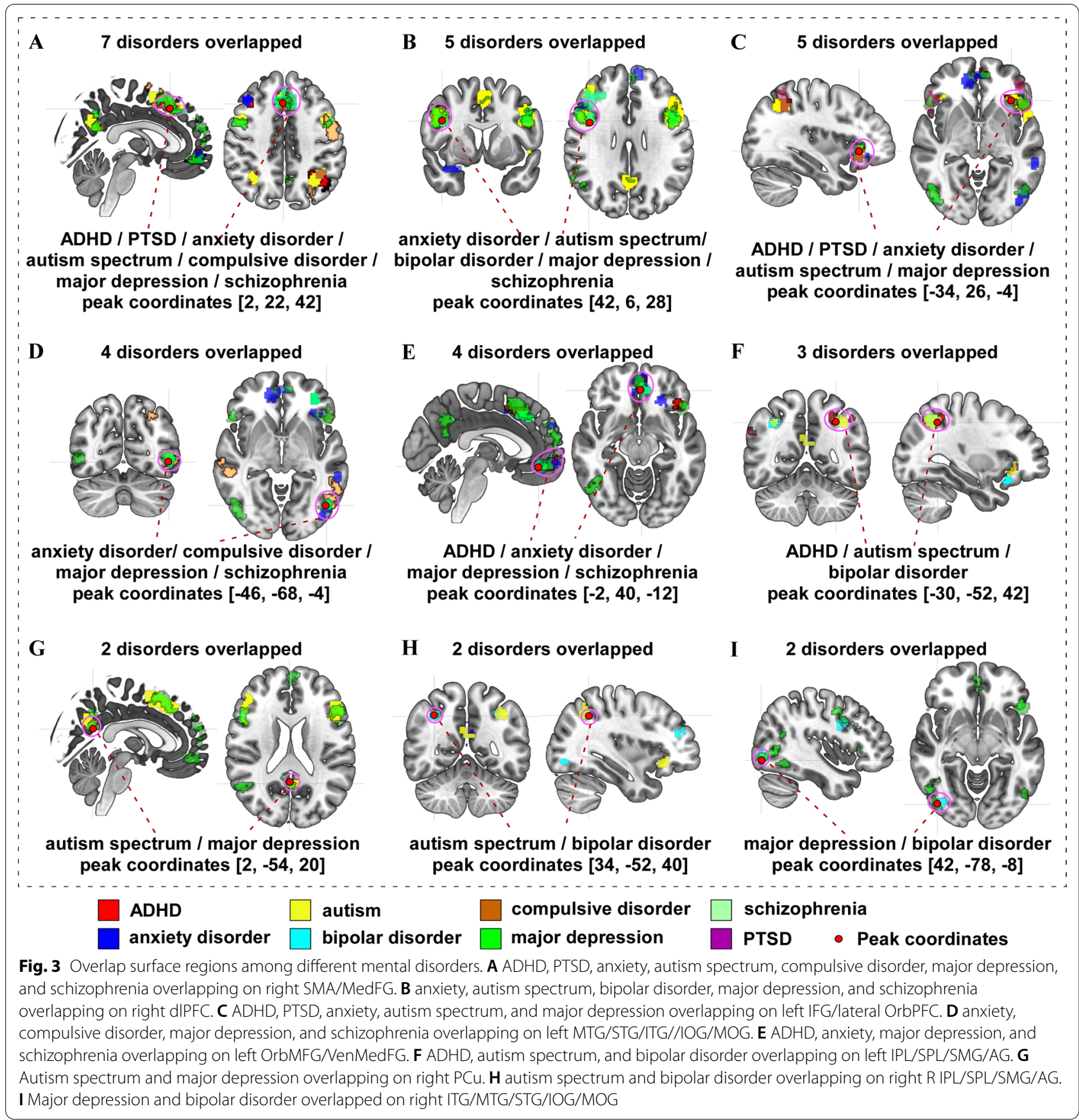

We believe that the target protocols may shed light on the development of scalp stimulation for the treatment of mental disorders.

Neurosynth is an automated tool that does not perform content analyses of how terms (each mental disorder) are being used in a literature, and all results such as activations/deactivations/connectivity/cerebral blood flow that appear in the paper will be included indiscriminately. Nevertheless, this does not prevent Neurosynth from supplying robust quantitative reverse inference data consistent with other databases and methods of analysis $[15,25]$. We believe this character/feature of Neurosynth will allow us to include all brain regions involved in brain pathophysiology of mental disorders. Lending support to this method's applicability, our findings are consistent with previous brain imaging studies on ADHD [26-28], anxiety disorder [29-31], autism spectrum disorder [32, $33]$, bipolar disorder $[34,35]$, compulsive disorder [36, 
37], major depression [38, 39], PTSD [40, 41], and schizophrenia [42-44].

Since the Neurosynth database contains a broad set of term-to-activation mappings, it will allow us to quantitatively identify emotional/pathological states from patterns of observed brain activity [15]. This however carries the problem of 'reverse inference', as most neuroimaging studies are designed to identify neural changes that result from known psychological manipulations or pathological status and not to determine what emotional/pathological state(s) a given pattern of activity implies, while the reverse inference requires knowledge of which brain regions/circuits are selectively, and not just consistently, associated with particular emotional/pathological states $[15,23,24]$.

\section{Overlap regions among different mental disorders}

Although each mental disorder is associated with different characteristic symptoms, the boundaries between mental disorders are complex and contentious. For example, schizophrenia, major depression, and bipolar disorder display overlapping clinical symptoms, particularly within the mood and psychosis spectrum [45, 46]. As different brain regions are involved in different specific functions, symptoms may be regarded as the causes or effects of dysfunction in certain regions. A large body of evidence suggests the brain regions and neuromodulatory systems involved in different mental disorders exhibit great overlap, and ultimate behavioral output circuits may be largely shared among disorders [47]. In this regard, identifying overlap regions associated with different mental disorders may be especially useful in delineating the etiology of mental disorders.

We found that the MedFG/SMA are involved in 7 disorders, including ADHD, PTSD, anxiety disorder, autism spectrum disorder, compulsive disorder, major depression, and schizophrenia. A large number of studies have revealed that the MedFG plays a crucial role in social cognition, an umbrella term for cognitive and emotional processes and abilities involved in effective interaction with the self and others, including representations of internal somatic states, knowledge about the self, perceptions of others and interpersonal motivations [48-51]. For instance, children with autism spectrum disorder (ASD) are characterized as having impairments in social communication and interaction as well as a range of stereotypic behaviors and language abilities [52]. Also, patients with ADHD become distracted because their focus on one object in the moment silences other, weaker messages. The frontal areas, especially the MedFG, are the most frequently reported regions of functional impairment in ASD and ADHD [53-56].
The SMA plays a key role in cognitive performance, especially in working memory. In a recent study, researchers found that damage to the SMA does not affect cognitive processes other than working memory, and as such, working memory impairment should be recognized as part of SMA syndrome [57]. Literature has suggested that working memory may be compromised in various mental disorders $[58,59]$. It has been particularly shown that anxiety constrains cognition by biasing attention toward the anticipation of threat, and that anxiety may reflect the highest level of normal motivational control in working memory [60, 61].

In addition, from the perspective of genetics, studies have suggested that approximately $80 \%$ of genetic syndromes currently known to be associated with ASD are characterized by motor impairments, which are closely related to SMA. These disturbances may be the earliest identifiable clinical abnormalities in ASD patients [62, 63].

We found that the right dIPFC is a notable region that is involved in 5 disorders (anxiety disorder, autism spectrum disorder, bipolar disorder, major depression, and schizophrenia). Literature suggests that the neural basis for emotion regulation deficits in mental disorders centers upon abnormalities within the fronto-limbic (cognitive-emotion) pathway $[64,65]$. Thus, normalization of the fronto-limbic pathway would be beneficial to emotional regulation. For instance, previous studies revealed that compared with healthy controls, adults and children with anxiety disorders demonstrated a decreased functional connectivity between the amygdala and frontal cortex, especially the dlPFC [66, 67], as well as an increased gray matter volume and decreased structural connectivity between these regions [4]. Also, the dlPFC is widely used as a target for anxiety disorder [68], autism spectrum disorder [69], bipolar disorder [70], major depression [8], and schizophrenia [7], and achieves significant clinical improvement.

We also found that anxiety disorder, bipolar disorder, compulsive disorder, major depression, and schizophrenia displayed overlap in the temporal and occipital regions. The temporal lobe has prominently been featured in studies of mental disorders due to its role in auditory and language processing. A study conducted by Anderson et al. revealed that various temporal lobe abnormalities cause the characteristic deficits in schizophrenia [71]. In addition, a previous study observed that patients with anxiety disorders demonstrate a significantly larger volume of white and grey matter in the STG [72]. Zhao and colleagues applied fMRI to investigate the activation and connectivity of the STG in patients with anxiety during different tasks and found that the activity of the STG increased during the silence task, while the 
functional connectivity decreased between the left and right STG during the threat-related task [73]. In another MRI study, researchers applied voxel-based morphometry analysis and a Likert-type scale, used to measure anxiety symptoms in 177 healthy individuals and found a positive correlation between the rating of anxiety symptoms and the grey matter volume in the prefrontal cortex and MTG [74], consistent with previous findings that these regions are involved in emotion regulation and are altered in patients with anxiety disorders $[75,76]$.

The occipital lobe contains most of the anatomical regions of the visual cortex and contributes to visual information processing, integration, and interpretation. Occipital lobe abnormalities have been detected in patients with certain mental disorders, including anxiety disorder, bipolar disorder, compulsive disorder, major depression, and schizophrenia [77-80]. These results are consistent with our findings from the current study. Take anxiety disorder for example, the anxious response and sensory-related fear are associated with regional instability of the occipital lobe, which plays a key role in emotional experience [80, 81]. A recent meta-analysis demonstrated that patients with anxiety disorders presented increased activation in the IOG [82]. Furthermore, anxiety patients, compared to healthy individuals, showed decreased activity in the SOG during both neutral and anxiety-inducing distractors in the working memory task [83], highlighting the principle role of the occipital lobe in emotional regulation and cognitive function.

Additionally, we found that ADHD, autism spectrum disorder, and bipolar disorder demonstrate overlaps in the parietal lobe, SMG, and AG. Literature has illustrated that ADHD, autism spectrum disorder, and bipolar disorder present with social cognitive dysfunction, which generally refers to abnormalities in mental operations underlying social interactions, including the perception and interpretation of intentions, dispositions, behaviors of others, and the generation of response to these behaviors [84-86]. These disorders present abnormalities in the parietal lobe, SMG, and AG, which play a critical role in social cognition, especially in learning abilities, language development and movement representation [87, 88]. In a recent neuroimaging study, researchers applied a graph-theoretic approach to investigate the organization of structural brain networks in adults with ADHD. They found that IPL, SMG, and AG were affected at the nodal level in relation to local efficiency and clustering. Lower local efficiency of SMG was associated with higher ADHD symptom scores, and lower local clustering of SMG correlated with ADHD symptom severity [89].

Finally, we found that autism spectrum disorder and major depression display an overlap on the $\mathrm{PCu}$. The
$\mathrm{PCu}$ is a key component of the default mode network (DMN), which is closely associated with social communication and interaction, as well as patterns of stereotypic and repetitive behaviors, and theory of mind; therefore, greatly contributing to various mental disorders [90-92]. In a recent meta-analysis of abnormal resting-state function connectivity (rsFC) in autism, Wang et al. observed a decreased resting-state brain activity in several DMN regions, including the $\mathrm{PCu}$ [93]. The result is consistent with a previous study, in which Jann et al. found decreased rsFC in the $\mathrm{PCu} /$ posterior cingulate cortex areas of the DMN in children with ASD [94]. Moreover, accumulating studies have highlighted the self-reflective role of the DMN involved in major depression $[95,96]$.

\section{Differences and similarities between the proposed neuroimaging-based target protocol and literature-documented stimulation targets}

Our target protocols are partly consistent with current prescriptions of scalp acupuncture or traditional acupuncture, and neuromodulation studies [2, 3, 9, 97]. In addition, our findings have extended previous knowledge on treatment of mental disorders with scalp stimulation method.

Take anxiety disorder as an example, the literature suggests that the middle line of the forehead (Ezhongxian, 1 cun long from Shenting [Governor Vessel \{GV\} 24] straight downward along the meridian), middle line of vertex (Dingzhongxian, from Baihui [GV 20] to Qianding [GV 21] along the midline of head), posterior temporal line (Niehouxian, from Shuaigu [GB 8] to Qubin [GB 7]), and upper-middle line of occiput (Zhenshang zhengzhongxian, from Qiangjian [GV 18] to Naohu [GV 17]) are widely recognized for alleviating anxiety using scalp acupuncture [2,98]. Overlapping regions exist in the comparison between the neuroimaging-based targets and the literature-documented targets, involving the frontal gyrus (SFG/MFG/MedFG/IFG), temporal gyrus (MTG/STG), and occipital gyrus (MOG/IOG). In addition, we also incorporated the dIPFC and the SMA in the current neuroimaging-based protocol.

In terms of neuromodulation interventions, a recent study summarized the stimulation targets of transcranial direct current stimulation in patients with an anxiety disorder, and found that 11 of the included research studies targeted the dIPFC in the treatment of anxiety [97]. In addition, transcranial alternating current stimulation on the dIPFC and the occipital cortex have been shown in several studies to reduce the severity of anxiety symptoms $[9,10]$. Furthermore, patients with anxiety disorders showed symptom remission after receiving repetitive transcranial magnetic stimulation (rTMS) on the dlPFC 
[14]. Most of the studies applying different neuromodulation techniques mainly used the dIPFC as the stimulation target for alleviating anxiety [9].

Nevertheless, our target protocol also includes additional brain areas such as the frontal gyrus (SFG/MFG/ MedFG/IFG), the SMA, and the temporal gyrus (MTG/ STG), which may expand the selection of potential targets in neuromodulation techniques for the treatment of anxiety.

We believe that the additional brain regions included in the neuroimaging-based targets reflect an enhanced understanding of the neural network involved in mental disorders and thus should be incorporated into the current scalp stimulation protocol. Further carefully designed and properly controlled studies are assuredly needed to evaluate the true potential of neuroimagingbased scalp stimulation targets.

\section{Limitations}

There are several limitations to our study. First, the keywords we used in searching the meta-analysis literature are the umbrella terms for the disorder; subtypes of the disorder are not included in this manuscript due to 1 ) each of the psychological disorders may be associated with multiple subtypes, and including these subtypes would considerably complicate the manuscript. 2) the literature on some subtypes may not be extensive enough to perform the meta-analysis. Further research on specific subtypes of these mental disorders is needed. Secondly, as our protocol is based on a brain imaging meta-analysis, clinical studies are needed to validate our findings. Additional functional and anatomical analyses, such as diffusion tensor imaging (DTI) and resting-state functional connectivity (rsFC), may further enhance the proposed protocols, particularly the individualized targets for each patient. Finally, medication information is not included in the Neurosynth database; thus, we cannot exclude the potential influence of medication. Further studies are needed to validate our finding on medicationfree patients.

Furthermore, the aim of this study was to explore the potential targets for mental disorders. Thus, our study may just represent one step of scalp stimulation protocols, the application and optimization of different treatment techniques (methods)/parameters/paradigms/field map to modulate these brain regions is beyond the scope of this manuscript. The optimal target selection for different scalp stimulation approaches to treat mental disorders could be an important future research endeavor. Additionally, to facilitate clinical application, we simplified the target protocol by including only eight or nine peak targets. Other brain regions may also play a critical role and should be applied in practice. Also, the locations of each target on the scalp are approximate, these locations may change when different parameters are applied or different studies are included in the analysis. Revision of the protocol will be needed as we advance our understanding of the brain physiology of mental disorders and scalp stimulation. Finally, to help the readers understand the brain function of identified areas, we have summarized the brain functions of each identified surface region associated with a corresponding mental disorder. It is worth noting that this summary may not be complete and accurate as we are still in the early stages of understanding the association between the brain and psychiatric disorders.

In summary, we have initiated an attempt to develop neuroimaging-based scalp stimulation target protocols for the treatment of eight common mental disorders. Our findings may facilitate the development and extend the clinical applications of scalp acupuncture, $\mathrm{tES}$, and other neuromodulation techniques for the treatment of mental disorders.

\section{Supplementary Information}

The online version contains supplementary material available at https://doi. org/10.1186/s12967-021-02993-1.

Additional file 1. Additional figures and tables

Acknowledgements

Not applicable.

Authors' contributions

Data analysis was performed by JC. JC, TCC-Z, YH, MNE, and JK prepared the manuscript draft. All authors read and approved the final manuscript.

\section{Funding}

Jian Kong was supported by the National Institutes of Health [Grant Numbers: R01 AT008563, R33 AT009310, R33 AT009341, R34 DA046635 (through the NIH HEAL Initiative), and R01 AG063975]. The content is solely the responsibility of the authors and does not necessarily represent the official views of the National Institutes of Health or its NIH HEAL Initiative.

Availability of data and materials

Data supporting the findings of this study are available from the corresponding author, upon reasonable request.

\section{Declarations}

Ethics approval and consent to participate Not applicable.

\section{Consent for publication}

Not applicable.

\section{Competing interests}

Jian Kong has a disclosure to report (holding equity in a startup company, MNT, and a patent to develop new peripheral neuromodulation devices). All other authors declare no conflicts of interest.

Received: 28 May 2021 Accepted: 17 July 2021

Published online: 10 August 2021 


\section{References}

1. Lu S. Scalp acupuncture therapy and its clinical application. J Tradit Chinese Med. 1991:11:272-80.

2. Pilkington K. Anxiety, depression and acupuncture: a review of the clinical research. Auton Neurosci Basic Clin. 2010;157:91-5. https://doi. org/10.1016/j.autneu.2010.04.002.

3. Tu CH, MacDonald I, Chen YH. The effects of acupuncture on glutamatergic neurotransmission in depression, anxiety, schizophrenia, and Alzheimer's disease: a review of the literature. Front Psychiatry. 2019;10:14.

4. Hilbert K, Lueken U, Beesdo-Baum K. Neural structures, functioning and connectivity in Generalized Anxiety Disorder and interaction with neuroendocrine systems: a systematic review. J Affect Disord. 2014:158:114-26.

5. Mochcovitch MD, Da Rocha Freire RC, Garcia RF, Nardi AE. A systematic review of $\mathrm{FMRI}$ studies in generalized anxiety disorder: evaluating its neural and cognitive basis. J Affect Disord. 2014;167:336-42.

6. Bremner JD. Brain imaging anxiety disorders. Expert Rev Neurother. 2004;4:275-84.

7. Brunoni AR, Shiozawa P, Truong D, et al. Understanding tDCS effects in schizophrenia: a systematic review of clinical data and an integrated computation modeling analysis. Expert Rev Med Devices. 2014;11:383-94.

8. Dunlop K, Hanlon CA, Downar J. Noninvasive brain stimulation treatments for addiction and major depression. Ann N Y Acad Sci. 2017;1394:31-54

9. Kar SK, Sarkar S. Neuro-stimulation techniques for the management of anxiety disorders: An update. Clin Psychopharmacol Neurosci. 2016:14:330-7.

10. Clancy KJ, Baisley SK, Albizu A, et al. Lasting connectivity increase and anxiety reduction via transcranial alternating current stimulation. Soc Cogn Affect Neurosci. 2018. https://doi.org/10.1093/scan/nsy096.

11. Clancy KJ, Baisley SK, Albizu A, et al. Transcranial alternating current stimulation induces long-term augmentation of neural connectivity and sustained anxiety reduction. BioRxiv. 2017. https://doi.org/10. 1101/204222.

12. Elyamany O, Leicht G, Herrmann CS, Mulert C. Transcranial alternating current stimulation (tACS): from basic mechanisms towards first applications in psychiatry. Eur Arch Psychiatry Clin Neurosci. 2021;271:135-56.

13. Shiozawa P, Leiva APG, Castro CDC, et al. Transcranial direct current stimulation for generalized anxiety disorder: a case study. Biol Psychiatry. 2014;75:e17-8.

14. Diefenbach GJ, Assaf M, Goethe JW, et al. Improvements in emotion regulation following repetitive transcranial magnetic stimulation for generalized anxiety disorder. J Anxiety Disord. 2016;43:1-7. https://doi. org/10.1016/j.janxdis.2016.07.002.

15. Yarkoni T, Poldrack RA, Nichols TE, et al. Large-scale automated synthesis of human functional neuroimaging data. Nat Methods. 2011:8:665-70. https://doi.org/10.1038/nmeth.1635.

16. Bystritsky A, Kaplan JT, Feusner JD, et al. A preliminary study of fMRIguided rTMS in the treatment of generalized anxiety disorder. J Clin Psychiatry. 2008;69:1092-8. https://doi.org/10.4088/JCP.v69n0708.

17. Blackmore J, Shrivastava S, Sallet J, et al. Ultrasound neuromodulation: a review of results, mechanisms and safety. Ultrasound Med Biol. 2019:45:1509-36.

18. Cao J, Huang Y, Meshberg N, et al. Neuroimaging-based scalp acupuncture locations for dementia. J Clin Med. 2020;9:2477. https://doi.org/10. 3390/jcm9082477.

19. Huang $Y$, Zhang B, Cao J, et al. Potential locations for noninvasive brain stimulation in treating autism spectrum disorders - a functional connectivity study. Front Psychiatry. 2020. https://doi.org/10.3389/fpsyt.2020. 00388.

20. Zhang B, Liu J, Bao T, et al. Locations for noninvasive brain stimulation in treating depressive disorders: a combination of meta-analysis and resting-state functional connectivity analysis. Aust New Zeal J Psychiatry. 2020. https://doi.org/10.1177/0004867420920372.

21. Liu J, Zhang B, Wilson G, Kong J. New perspective for non-invasive brain stimulation site selection in mild cognitive impairment: based on metaand functional connectivity analyses. Front Aging Neurosci. 2019;11:228 https://doi.org/10.3389/fnagi.2019.00228.
22. Cutini S, Scatturin P, Zorzi M. A new method based on ICBM152 head surface for probe placement in multichannel fNIRS. Neuroimage. 2011:54:919-27. https://doi.org/10.1016/j.neuroimage.2010.09.030

23. Td W, Ma L, Te N, et al. Evaluating the consistency and specificity of neuroimaging data using meta-analysis. Neuroimage. 2009. https://doi.org/ 10.1016/J.NEUROIMAGE.2008.10.061.

24. RA $P$, . Can cognitive processes be inferred from neuroimaging data? Trends Cogn Sci. 2006;10:59-63. https://doi.org/10.1016/J.TICS.2005.12. 004.

25. Genon S, Reid A, Langner R, et al. How to characterize the function of a brain region. Trends Cogn Sci. 2018;22:350-64.

26. Rubia K. Neuro-anatomic evidence for the maturational delay hypothesis of ADHD. Proc Natl Acad Sci USA. 2007;104:19663-4.

27. Krain AL, Castellanos FX. Brain development and ADHD. Clin Psychol Rev. 2006;26:433-44. https://doi.org/10.1016/j.cpr.2006.01.005.

28. Shaw P, Eckstrand K, Sharp W, et al. Attention-deficit/hyperactivity disorder is characterized by a delay in cortical maturation. Proc Natl Acad Sci USA. 2007;104:19649-54. https://doi.org/10.1073/pnas.0707741104.

29. Tovote P, Fadok JP, Lüthi A. Neuronal circuits for fear and anxiety. Nat Rev Neurosci. 2015;16:317-31.

30. Felger JC. Imaging the role of inflammation in mood and anxiety-related disorders. Curr Neuropharmacol. 2017;15:533. https://doi.org/10.2174/ $1570159 \times 15666171123201142$

31. Park J, Moghaddam B. Impact of anxiety on prefrontal cortex encoding of cognitive flexibility. Neuroscience. 2017;345:193-202.

32. Robertson CE, Baron-Cohen S. Sensory perception in autism. Nat Rev Neurosci. 2017:18:671-84

33. Zürcher NR, Bhanot A, McDougle CJ, Hooker JM. A systematic review of molecular imaging (PET and SPECT) in autism spectrum disorder: current state and future research opportunities. Neurosci Biobehav Rev. 2015:52:56-73.

34. Phillips ML, Ladouceur CD, Drevets WC. A neural model of voluntary and automatic emotion regulation: implications for understanding the pathophysiology and neurodevelopment of bipolar disorder. Mol Psychiatry. 2008;13:833-57.

35. Ashok AH, Marques TR, Jauhar S, et al. The dopamine hypothesis of bipolar affective disorder: the state of the art and implications for treatment. Mol Psychiatry. 2017;22:666-79.

36. Nakao T, Okada K, Kanba S. Neurobiological model of obsessive-compulsive disorder: evidence from recent neuropsychological and neuroimaging findings. Psychiatry Clin Neurosci. 2014;68:587-605. https://doi.org/ 10.1111/pen.12195.

37. Piras F, Piras F, Caltagirone C, Spalletta G. Brain circuitries of obsessive compulsive disorder: a systematic review and meta-analysis of diffusion tensor imaging studies. Neurosci Biobehav Rev. 2013;37:2856-77.

38. Koolschijn PCMP, Van Haren NEM, Lensvelt-Mulders GJLM, et al. Brain volume abnormalities in major depressive disorder: a meta-analysis of magnetic resonance imaging studies. Hum Brain Mapp. 2009;30:3719-35. https://doi.org/10.1002/hbm.20801.

39. Arnone D, Job D, Selvaraj S, et al. Computational meta-analysis of statistical parametric maps in major depression. Hum Brain Mapp. 2016;37:1393-404. https://doi.org/10.1002/hbm.23108.

40. Kühn S, Gallinat J. Gray matter correlates of posttraumatic stress disorder: a quantitative meta-analysis. Biol Psychiatry. 2013;73:70-4. https://doi. org/10.1016/j.biopsych.2012.06.029.

41. Pitman RK, Rasmusson AM, Koenen KC, et al. Biological studies of posttraumatic stress disorder. Nat Rev Neurosci. 2012;13:769-87.

42. Hu ML, Zong XF, Mann JJ, et al. A review of the functional and anatomical default mode network in schizophrenia. Neurosci Bull. 2017;33:73-84

43. Wojtalik JA, Smith MJ, Keshavan MS, Eack SM. A systematic and metaanalytic review of neural correlates of functional outcome in schizophrenia. Schizophr Bull. 2017:43:1329-47.

44. Molent C, Olivo D, Wolf RC, et al. Functional neuroimaging in treatment resistant schizophrenia: a systematic review. Neurosci Biobehav Rev. 2019;104:178-90.

45. Pearlson GD. Etiologic, phenomenologic, and endophenotypic overlap of schizophrenia and bipolar disorder. Annu Rev Clin Psychol. 2015;11:25181. https://doi.org/10.1146/annurev-clinpsy-032814-112915.

46. Kempf L, Hussain N, Potash JB. Mood disorder with psychotic features, schizoaffective disorder, and schizophrenia with mood features: trouble at the borders. Int Rev Psychiatry. 2005;17:9-19. 
47. Goodwin GM. The overlap between anxiety, depression, and obsessivecompulsive disorder. Dialogues Clin Neurosci. 2015;17:249-60. https:// doi.org/10.31887/dens.2015.17.3/ggoodwin.

48. Coricelli G, Critchley HD, Joffily M, et al. Regret and its avoidance: a neuroimaging study of choice behavior. Nat Neurosci. 2005;8:1255-62. https:// doi.org/10.1038/nn1514.

49. Amodio DM, Frith CD. Meeting of minds: the medial frontal cortex and social cognition. Nat Rev Neurosci. 2006;7:268-77.

50. Knutson B, Taylor J, Kaufman M, et al. Distributed neural representation of expected value. J Neurosci. 2005;25:4806-12. https://doi.org/10.1523/ JNEUROSCI.0642-05.2005.

51. Walton ME, Devlin JT, Rushworth MFS. Interactions between decision making and performance monitoring within prefrontal cortex. Nat Neurosci. 2004;7:1259-65. https://doi.org/10.1038/nn1339.

52. Tager-Flusberg $\mathrm{H}$, Joseph R, Folstein S. Current directions in research on autism. Ment Retard Dev Disabil Res Rev. 2001;7:21-9.

53. Danielson NB, Guo JN, Blumenfeld $H$. The default mode network and altered consciousness in epilepsy. Behav Neurol. 2011;24:55-65. https:// doi.org/10.3233/BEN-2011-0310.

54. Cubillo A, Halari R, Ecker C, et al. Reduced activation and inter-regional functional connectivity of fronto-striatal networks in adults with childhood Attention-Deficit Hyperactivity Disorder (ADHD) and persisting symptoms during tasks of motor inhibition and cognitive switching. J Psychiatr Res. 2010;44:629-39. https://doi.org/10.1016/j.jpsychires.2009. 11.016.

55. Bush G. Attention-deficit/hyperactivity disorder and attention networks. Neuropsychopharmacology. 2010;35:278-300.

56. Mundy P. A review of joint attention and social-cognitive brain systems in typical development and autism spectrum disorder. Eur J Neurosci. 2018;47:497-514. https://doi.org/10.1111/ejn.13720.

57. Cañas $A$, Juncadella $M$, Lau R, et al. Working memory deficits after lesions involving the supplementary motor area. Front Psychol. 2018;9:765. https://doi.org/10.3389/fpsyg.2018.00765.

58. Schweitzer JB, Faber TL, Grafton ST, et al. Alterations in the functional anatomy of working memory in adult attention deficit hyperactivity disorder. Am J Psychiatry. 2000;157:278-80. https://doi.org/10.1176/appi. ajp.157.2.278.

59. Ansari S. The therapeutic potential of working memory training for treating mental disorders. Front Hum Neurosci. 2015;9:1-3. https://doi.org/10. 3389/fnhum.2015.00481.

60. Luu P, Tucker DM, Derryberry D. Anxiety and the motivational basis of working memory. Cognit Ther Res. 1998;22:577-94.

61. Lukasik KM, Waris $O$, Soveri A, et al. The relationship of anxiety and stress with working memory performance in a large non-depressed sample. Front Psychol. 2019. https://doi.org/10.3389/fpsyg.2019.00004.

62. Abrahams BS, Geschwind DH. Connecting genes to brain in the autism spectrum disorders. Arch Neurol. 2010;67:395-9.

63. Bryson SE, Zwaigenbaum L, Brian J, et al. A prospective case series of high-risk infants who developed autism. J Autism Dev Disord. 2007;37:12-24. https://doi.org/10.1007/s10803-006-0328-2.

64. Shackman AJ, Fox AS, Seminowicz DA (2015) The cognitive-emotional brain: Opportunitvnies and challenges for understanding neuropsychiatric disorders. Behav. Brain Sci. 38:e86

65. Lima IMM, Peckham AD, Johnson SL. Cognitive deficits in bipolar disorders: implications for emotion. Clin Psychol Rev. 2018;59:126-36.

66. Jung $\mathrm{Y}-\mathrm{H}$, Shin JE, Lee $\mathrm{Yl}$, et al. Altered amygdala resting-state functional connectivity and hemispheric asymmetry in patients with social anxiety disorder. Front Psychiatry. 2018;9:164. https://doi.org/10.3389/fpsyt.2018. 00164.

67. Makovac E, Meeten F, Watson DR, et al. Alterations in amygdala-prefrontal functional connectivity account for excessive worry and autonomic dysregulation in generalized anxiety disorder. Biol Psychiatry. 2016;80:78695. https://doi.org/10.1016/j.biopsych.2015.10.013.

68. Dilkov D, Hawken ER, Kaludiev E, Milev R. Repetitive transcranial magnetic stimulation of the right dorsal lateral prefrontal cortex in the treatment of generalized anxiety disorder: a randomized, double-blind sham controlled clinical trial. Prog Neuro-Psychopharmacol Biol Psychiatry. 2017;78:61-5. https://doi.org/10.1016/j.pnpbp.2017.05.018.

69. Hadoush H, Nazzal M, Almasri NA, et al. Therapeutic effects of bilateral anodal transcranial direct current stimulation on prefrontal and motor cortical areas in children with autism spectrum disorders: a pilot study. Autism Res. 2020;13:828-36. https://doi.org/10.1002/aur.2290.

70. Brunoni AR, Ferrucci R, Bortolomasi M, et al. Transcranial direct current stimulation (tDCS) in unipolar vs. bipolar depressive disorder. Prog NeuroPsychopharmacol Biol Psychiatry. 2011;35:96-101. https://doi.org/10. 1016/j.pnpbp.2010.09.010.

71. Anderson JE, Wible CG, McCarley RW, et al. An MRI study of temporal lobe abnormalities and negative symptoms in chronic schizophrenia. Schizophr Res. 2002;58:123-34. https://doi.org/10.1016/S0920-9964(01)00372-3.

72. De Bellis MD, Keshavan MS, Shifflett H, et al. Superior temporal gyrus volumes in pediatric generalized anxiety disorder. Biol Psychiatry. 2002;51:553-62. https://doi.org/10.1016/S0006-3223(01)01375-0.

73. Zhao X, Xi Q, Wang P, et al. Altered activity and functional connectivity of superior temporal gyri in anxiety disorders: a functional magnetic resonance imaging study. Korean J Radiol. 2014;15:523-9. https://doi.org/ 10.3348/kjr.2014.15.4.523.

74. Besteher B, Gaser C, Langbein K, et al. Effects of subclinical depression, anxiety and somatization on brain structure in healthy subjects. J Affect Disord. 2017;215:111-7. https://doi.org/10.1016/j.jad.2017.03.039.

75. Strawn JR, Wehry AM, Chu WJ, et al. Neuroanatomic abnormalities in adolescents with generalized anxiety disorder: a voxel-based morphometry study. Depress Anxiety. 2013;30:842-8. https://doi.org/10.1002/da.22089.

76. Shang J, Fu Y, Ren Z, et al. The common traits of the ACC and PFC in anxiety disorders in the DSM-5: Meta-analysis of voxel-based morphometry studies. PLoS ONE. 2014. https://doi.org/10.1371/journal.pone.0093432.

77. Falkai P, Schneider T, Greve B, et al. Reduced frontal and occipital lobe asymmetry on the CT-scans of schizophrenic patients. Its specificity and clinical significance. J Neural Transm. 1995;99:63-77. https://doi.org/10. 1007/BF01271470.

78. Abé C, Rolstad S, Petrovic P, et al. Bipolar disorder type I and II show distinct relationships between cortical thickness and executive function. Acta Psychiatr Scand. 2018;138:325-35. https://doi.org/10.1111/acps. 12922.

79. Chen Y, Meng Z, Zhang Z, et al. The right thalamic glutamate level correlates with functional connectivity with right dorsal anterior cingulate cortex/middle occipital gyrus in unmedicated obsessive-compulsive disorder: a combined fMRI and $1 \mathrm{H}-\mathrm{MRS}$ study. Aust N Z J Psychiatry. 2019;53:207-18. https://doi.org/10.1177/0004867418806370.

80. Paquette $V$, Lévesque J, Mensour B, et al. "Change the mind and you change the brain": effects of cognitive-behavioral therapy on the neural correlates of spider phobia. Neuroimage. 2003;18:401-9. https://doi.org/ 10.1016/S1053-8119(02)00030-7.

81. Lai CH, Te WuY. Decreased regional homogeneity in lingual gyrus, increased regional homogeneity in cuneus and correlations with panic symptom severity of first-episode, medication-naïve and late-onset panic disorder patients. Psychiatry Res. 2013;211:127-31. https://doi.org/10. 1016/j.pscychresns.2012.11.006.

82. Picó-Pérez M, Radua J, Steward T, et al. Emotion regulation in mood and anxiety disorders: a meta-analysis of fMRI cognitive reappraisal studies. Prog Neuro-Psychopharmacol Biol Psychiatry. 2017;79:96-104.

83. Moon C-M, Jeong G-W. Functional neuroanatomy on the working memory under emotional distraction in patients with generalized anxiety disorder. Psychiatry Clin Neurosci. 2015;69:609-19. https://doi.org/10. 1111/pcn.12295.

84. Stefanik L, Erdman L, Ameis SH, et al. Brain-behavior participant similarity networks among youth and emerging adults with schizophrenia spectrum, autism spectrum, or bipolar disorder and matched controls. Neuropsychopharmacology. 2018;43:1180-8. https://doi.org/10.1038/ npp.2017.274.

85. Martins FE, Sanvicente-Vieira B, Grassi-Oliveira R, Brietzke E. Social cognition and theory of mind: controversies and promises for understanding major psychiatric disorders. Psychol Neurosci. 2011;4:347-51. https://doi. org/10.3922/j.psns.2011.3.008.

86. Millan MJ, Agid Y, Brüne M, et al. Cognitive dysfunction in psychiatric disorders: characteristics, causes and the quest for improved therapy. Nat Rev Drug Discov. 2012;11:141-68.

87. Arbib MA. From monkey-like action recognition to human language: an evolutionary framework for neurolinguistics. Behav Brain Sci. 2005;28:105-24. https://doi.org/10.1017/S0140525X05000038.

88. McClain M, Foundas A. Apraxia. Curr Neurol Neurosci Rep. 2004;4:471-6. 
89. Sidlauskaite J, Caeyenberghs K, Sonuga-Barke E, et al. Whole-brain structural topology in adult attention-deficit/hyperactivity disorder: preserved global - Disturbed local network organization. Neurolmage Clin. 2015;9:506-12. https://doi.org/10.1016/j.nicl.2015.10.001.

90. Washington SD, Gordon EM, Brar J, et al. Dysmaturation of the default mode network in autism. Hum Brain Mapp. 2014;35:1284-96. https://doi. org/10.1002/hbm.22252.

91. Rudie JD, Hernandez LM, Brown JA, et al. Autism-associated promoter variant in MET impacts functional and structural brain networks. Neuron. 2012;75:904-15. https://doi.org/10.1016/j.neuron.2012.07.010.

92. Buckner RL, Andrews-Hanna JR, Schacter DL. The brain's default network: anatomy, function, and relevance to disease. Ann NY Acad Sci. 2008;1124:1-38. https://doi.org/10.1196/annals.1440.011.

93. Wang W, Liu J, Shi S, et al. Altered resting-state functional activity in patients with autism spectrum disorder: a quantitative meta-analysis. Front Neurol. 2018;9:556. https://doi.org/10.3389/fneur.2018.00556.

94. Jann K, Hernandez LM, Beck-Pancer D, et al. Altered resting perfusion and functional connectivity of default mode network in youth with autism spectrum disorder. Brain Behav. 2015. https://doi.org/10.1002/brb3.358.
95. Li B, Liu L, Friston KJ, et al. A treatment-resistant default mode subnetwork in major depression. Biol Psychiatry. 2013;74:48-54. https://doi.org/ 10.1016/j.biopsych.2012.11.007.

96. Sestieri C, Corbetta M, Romani GL, Shulman GL. Episodic memory retrieval, parietal cortex, and the default mode network: functional and topographic analyses. J Neurosci. 2011;31:4407-20. https://doi.org/10. 1523/JNEUROSCI.3335-10.2011.

97. Stein DJ, Medeiros LF, Caumo W, Torres ILS. Transcranial direct current stimulation in patients with anxiety: current perspectives. Neuropsychiatr Dis Treat. 2020;16:161-9.

98. Dong G, Cao D, Dong Y, et al. Scalp acupuncture for sleep disorder induced by pre-examination anxiety in undergraduates. World J Acupunct - Moxibustion. 2018;28:156-60. https://doi.org/10.1016/j.wjam. 2018.09.003.

\section{Publisher's Note}

Springer Nature remains neutral with regard to jurisdictional claims in published maps and institutional affiliations.
Ready to submit your research? Choose BMC and benefit from:

- fast, convenient online submission

- thorough peer review by experienced researchers in your field

- rapid publication on acceptance

- support for research data, including large and complex data types

- gold Open Access which fosters wider collaboration and increased citations

- maximum visibility for your research: over 100M website views per year

At BMC, research is always in progress.

Learn more biomedcentral.com/submissions 